ESAIM: M2AN 49 (2015) 349-372

DOI: $10.1051 / \mathrm{m} 2 \mathrm{an} / 2014035$
ESAIM: Mathematical Modelling and Numerical Analysis

www.esaim-m2an.org

\title{
CONVERGENCE OF THE CELL AVERAGE TECHNIQUE FOR SMOLUCHOWSKI COAGULATION EQUATION
}

\author{
AnkiK Kumar Giri ${ }^{1,2}$ And Atulya K. NAGAR ${ }^{3}$
}

\begin{abstract}
We present the convergence analysis of the cell average technique, introduced in [J. Kumar et al., Powder Technol. 179 (2007) 205-228.], to solve the nonlinear continuous Smoluchowski coagulation equation. It is shown that the technique is second order accurate on uniform grids and first order accurate on non-uniform smooth (geometric) grids. As an essential ingredient, the consistency of the technique is thoroughly discussed.
\end{abstract}

Mathematics Subject Classification. 45J05, 45K05, 45L05, 65R20.

Received December 15, 2012. Revised March 19, 2014.

Published online February 19, 2015.

\section{INTRODUCTION}

In this article we study some mathematical issues related to the convergence of the cell average technique (CAT) [19] for solving the continuous Smoluchowski coagulation equation (SCE) which describes the dynamic evolution of particle growth. This model has many applications in biology, polymer science, astrophysics and oil industry etc. The nonlinear continuous SCE reads as

$$
\frac{\partial f(t, x)}{\partial t}=\frac{1}{2} \int_{0}^{x} \beta(x-y, y) f(t, x-y) f(t, y) \mathrm{d} y-\int_{0}^{\infty} \beta(x, y) f(t, x) f(t, y) \mathrm{d} y,
$$

with

$$
\left.f(x, 0)=f^{\text {in }}(x) \geq 0, \quad x \in\right] 0, \infty[.
$$

Here the number density of particles of volume $x>0$ at time $t \geq 0$ is denoted by $f(x, t) \geq 0$. The coagulation kernel $\beta(x, y) \geq 0$ represents the rate at which particles of volume $x$ coalesce with particles of volume $y$. It will be assumed throughout the article that $\beta(x, y)=\beta(y, x)$ for all $x, y>0$, i.e. symmetric and $\beta(x, y)=0$ for either $x=0$ or $y=0$. The integrals on the right-hand side of (1.1) represent, respectively,

- birth of particles of volume $x$ as a result of coagulation events of particles with volumes $y$ and $x-y$ $(0 \leq y \leq x)$

\footnotetext{
Keywords and phrases. Particles, coagulation, cell average technique, consistency, Lipschitz condition, convergence.

1 Department of Mathematics, Indian Institute of Technology Roorkee, Roorkee 247667, Uttarakhand, India.

2 Johann Radon Institute for Computational and Applied Mathematics (RICAM), Austrian Academy of Sciences, Altenbergerstrasse 69, 4040 Linz, Austria. ankik.giri@ricam.oeaw.ac.at; ankik.math@gmail.com; ankikgiri.fma@iitr.ac.in

3 Department of Mathematics and computer science, Liverpool Hope University, Hope Park, Liverpool, UK.
} 
- death of particles of volume $x$ due to the coagulation events with particles of volume $y(0 \leq y<\infty)$.

There are several results available on the existence and uniqueness of solutions to (1.1), see e.g. $[2,4,7,15-17,25-27,30,31,35]$. To show all these results, one always needs certain growth conditions on coagulation kernels. The SCE (1.1) is analytically solvable only for some specific examples of coagulation kernels, see $[3,11,12]$. Because of these restrictions, several researchers are always interested to solve such models by applying different numerical techniques with a detailed study of their mathematical analysis.

A large variety of numerical methods have been applied for solving SCE: finite element methods $[8,32,33]$, finite volume methods [1,9,10], stochastic methods [5,6,28], moment methods [34] and scetional methods [19-22]. Stochastic methods are very powerful to solve such problems. Otherwise, most of these methods may give a good approximation of number density but a poor approximation of moments. However, the moment methods approximate very accurately the moments of the number density but are unable to give a precise information about the number density. Among all these methods for solving SCE (1.1), the sectional methods have become very popular because they not only approximate accurately some selected moments but also give satisfactory results for the number density.

Among all sectional methods, the fixed pivot technique (FPT) [21] is most widely used method in the literature. In the FPT, each new born particle, which is not positioned at a pivot point of any cell has to be assigned onto the neighboring pivot points. A step to improve the existing sectional methods has been made in $[19,20]$ as the cell average technique (CAT). Unlike the FPT, here in the CAT, the average of all new born particles in a cell is assigned to the neighboring pivot points. In both of the methods, the reassignment is done in such a way that the total number and mass remain conserved. For solving the linear breakage/fragmentation equation, both FPT and CAT, in [23,24], are shown second order accurate on uniform and non-uniform smooth meshes. Recently, in [14], it is shown that the FPT is second order accurate on uniform and non-uniform smooth grids for solving the nonlinear SCE.

The purpose of this work is to demonstrate the convergence analysis of CAT for solving SCE (1.2) on uniform and non-uniform smooth geometric grids. To the best of our knowledge, this is the first attempt to show the convergence of CAT for solving nonlinear continuous SCE. The work presented here is motivated from [14,24].

To apply a numerical method, first we need to consider the following truncated form of the problem (1.1) by taking a finite computational domain $] 0, R]$ where $0<R<\infty$.

$$
\frac{\partial n(t, x)}{\partial t}=\frac{1}{2} \int_{0}^{x} \beta(x-y, y) n(t, x-y) n(t, y) \mathrm{d} y-\int_{0}^{R} \beta(x, y) n(t, x) n(t, y) \mathrm{d} y,
$$

with

$$
\left.\left.n(x, 0)=n^{\text {in }}(x) \geq 0, \quad x \in \Omega:=\right] 0, R\right],
$$

where $n(t, x)$ represents the solution to the truncated equation (1.2). The existence and uniqueness of nonnegative solutions for the truncated SCE $(1.2)$ has been shown in $[4,35]$. In $[4,7,15,16,26,35]$, it is proven that the sequence of solutions to the truncated problems converge weakly to the solution of the original problem in a weighted $L^{1}$ space as $R \rightarrow \infty$ for certain classes of kernels.

The plan of this paper is as follows. The mathematical formulation of CAT is recalled in the next section. The main convergence result is stated as Theorem 2.6 at the end of Section 2. In order to prove the main result, the consistency of the method and Lipschitz conditions are investigated in Sections 3 and 4, respectively. Finally, some conclusions are made in Section 5.

\section{The CELl AVERAge technique}

The cell average technique approximates the total number of particles in finite number of cells. As a first step, the continuous interval $\Omega:=] 0, R]$ is divided into a small number of cells defining size classes

$$
\left.\left.\Lambda_{i}:=\right] x_{i-1 / 2}, x_{i+1 / 2}\right], i=1, \ldots, I,
$$


with

$$
x_{1 / 2}=0, \quad x_{I+1 / 2}=R .
$$

The representative of each size class, usually the center point of each cell $x_{i}=\left(x_{i-1 / 2}+x_{i+1 / 2}\right) / 2$, is called pivot or grid point. We introduce $\Delta x_{\min }$ and $\left.\Delta x \in\right] 0,1[$ to satisfy

$$
\Delta x_{\min } \leq \Delta x_{i}=x_{i+1 / 2}-x_{i-1 / 2} \leq \Delta x .
$$

For the purpose of later analysis, we assume that there exists a positive constant $K$ (independent of grid) such that

$$
\frac{\Delta x}{\Delta x_{\min }} \leq K \quad \text { (quasi uniformity) }
$$

The total number of particles in the $i$ th cell is given as

$$
N_{i}(t)=\int_{x_{i-1 / 2}}^{x_{i+1 / 2}} n(t, x) \mathrm{d} x .
$$

Integrating the continuous equation (1.2) over the $i$ th cell we obtain

$$
\frac{\mathrm{d} N_{i}(t)}{\mathrm{d} t}=B_{i}-D_{i}, \quad i=1, \ldots, I .
$$

The total birth rate $B_{i}$ and the death rate $D_{i}$ are given as

$$
B_{i}=\frac{1}{2} \int_{x_{i-1 / 2}}^{x_{i+1 / 2}} \int_{0}^{x} \beta(x-y, y) n(t, x-y) n(t, y) \mathrm{d} y \mathrm{~d} x
$$

and

$$
D_{i}=\int_{x_{i-1 / 2}}^{x_{i+1 / 2}} \int_{0}^{x_{I+1 / 2}} \beta(x, y) n(t, y) n(t, x) \mathrm{d} y \mathrm{~d} x .
$$

The above equations yield a semi-discrete system in $\mathbb{R}^{I}$

$$
\frac{d \mathbf{N}}{\mathrm{d} t}=\mathbf{B}-\mathbf{D}, \quad \text { with } \mathbf{N}(0)=\mathbf{N}^{\text {in }},
$$

where $\mathbf{N}, \mathbf{B}, \mathbf{D} \in \mathbb{R}^{I}$. The $i$ th component of vectors $\mathbf{N}, \mathbf{B}$, and $\mathbf{D}$ are respectively defined in (2.2)-(2.4). The vector $\mathbf{N}$ is formed by the vector of values of the step function obtained by $L^{2}$ projection of the exact solution $n$ into the space of step functions, which are constant on each cell. Note that this projection error can easily be shown of second order, see [13]. The total discrete birth and death rates of particles are evaluated by substituting the number density approximation

$$
n(t, x) \approx \sum_{i=1}^{I} N_{i}(t) \delta\left(x-x_{i}\right)
$$

into equations (2.3) and (2.4) as

$$
\hat{B}_{i}=\sum_{x_{i-1 / 2} \leq x_{j}+x_{k}<x_{i+1 / 2}}^{j \geq k}\left(1-\frac{1}{2} \delta_{j, k}\right) \beta\left(x_{k}, x_{j}\right) N_{j}(t) N_{k}(t),
$$


and

$$
\hat{D}_{i}=N_{i}(t) \sum_{j=1}^{I} \beta\left(x_{i}, x_{j}\right) N_{j}(t)
$$

Here $\hat{B}_{i}$ and $\hat{D}_{i}$ denote the discrete birth and death rates, respectively, in the $i$ th cell. The total volume flux $V_{i}$ into cell $i$ as a result of aggregation is given by

$$
V_{i}=\frac{1}{2} \int_{x_{i-1 / 2}}^{x_{i+1 / 2}} \int_{0}^{x} x \beta(x-y, y) n(t, x-y) n(t, y) \mathrm{d} y \mathrm{~d} x .
$$

Similarly to the discrete birth rate the discrete volume flux can be obtained as

$$
\hat{V}_{i}=\sum_{x_{i-1 / 2} \leq x_{j}+x_{k}<x_{i+1 / 2}}^{j \geq k}\left(1-\frac{1}{2} \delta_{j, k}\right) \beta\left(x_{k}, x_{j}\right) N_{j}(t) N_{k}(t)\left(x_{j}+x_{k}\right) .
$$

Consequently, the average volume $\bar{v}_{i} \in\left[x_{i-1 / 2}, x_{i+1 / 2}\right]$ of all new born particles in the $i$ th cell can be evaluated as

$$
\bar{v}_{i}=\frac{\hat{V}_{i}}{\hat{B}_{i}}, \quad \hat{B}_{i}>0
$$

We do not need volume average $\bar{v}_{i}$ in case of $\hat{B}_{i}=0$. However, for $\hat{B}_{i}=0$, we can fix $\bar{v}_{i}=x_{i}$. The main idea of the scheme is to assign temporarily all new born particles in the $i$ th cell to the average volume $\bar{v}_{i}$. If the average volume $\bar{v}_{i}$ is same as the pivot point $x_{i}$ then the total birth $\hat{B}_{i}$ of the new born particles can be assigned to the pivot $x_{i}$ only. But this is rarely possible, and hence, the total birth $\hat{B}_{i}$ has to be assigned to the neighboring pivots in such a way that the total number and mass remain conserved during this reassignment. Finally, the resultant set of ODEs takes the following form

$$
\frac{\mathrm{d} \hat{N}_{i}}{\mathrm{~d} t}=\hat{B}_{i}^{C A}-\hat{D}_{i}^{C A}
$$

The above discretized system can also be written in the following vector form

$$
\frac{\mathrm{d} \hat{\mathbf{N}}}{\mathrm{d} t}=\hat{\mathbf{B}}(\hat{\mathbf{N}})-\hat{\mathbf{D}}(\hat{\mathbf{N}})=: \hat{\mathbf{F}}(t, \hat{\mathbf{N}}), \text { with } \hat{\mathbf{N}}(0)=\mathbf{N}^{\text {in }},
$$

where $\hat{\mathbf{N}}, \hat{\mathbf{B}}, \hat{\mathbf{D}} \in \mathbb{R}^{I}$. The numerical approximation of total number of particles in $i$ th cell, $N_{i}(t)$, is defined by $\hat{N}_{i}(t)$ which is the $i$ th component of the vector $\hat{\mathbf{N}}$. The discretized birth term, $\hat{B}_{i}^{C A}$, and death term, $\hat{D}_{i}^{C A}$, obtained from the cell average technique are defined below. These are the $i$ th components of the vectors $\hat{\mathbf{B}}$ and $\hat{\mathbf{D}}$ respectively. Let us consider the Heaviside function

$$
H(x):= \begin{cases}1 & \text { if } x>0 \\ \frac{1}{2} & \text { if } x=0 \\ 0 & \text { if } x<0\end{cases}
$$

and

$$
\lambda_{i}^{ \pm}(x)=\frac{x-x_{i \pm 1}}{x_{i}-x_{i \pm 1}}
$$


Then the birth and death terms are given as

$$
\begin{aligned}
\hat{B}_{i}^{C A}:= & \hat{B}_{i-1} \lambda_{i}^{-}\left(\bar{v}_{i-1}\right) H\left(\bar{v}_{i-1}-x_{i-1}\right)+\hat{B}_{i} \lambda_{i}^{+}\left(\bar{v}_{i}\right) H\left(\bar{v}_{i}-x_{i}\right) \\
& +\hat{B}_{i} \lambda_{i}^{-}\left(\bar{v}_{i}\right) H\left(x_{i}-\bar{v}_{i}\right)+\hat{B}_{i+1} \lambda_{i}^{+}\left(\bar{v}_{i+1}\right) H\left(x_{i+1}-\bar{v}_{i+1}\right),
\end{aligned}
$$

and

$$
\hat{D}_{i}^{C A}:=\hat{D}_{i}=N_{i}(t) \sum_{j=1}^{I} \beta\left(x_{i}, x_{j}\right) N_{j}(t) .
$$

The first and the fourth terms on the right hand side of equation (2.15) can be set to zero for $i=1$ and $i=I$, respectively. The detailed formulation can be found in [19].

By using (2.6) and (2.7) the cell average technique (2.11) can be written as

$$
\begin{aligned}
\frac{\mathrm{d} \hat{N}_{i}(t)}{\mathrm{d} t}= & \lambda_{i}^{-}\left(\bar{v}_{i-1}\right) H\left(\bar{v}_{i-1}-x_{i-1}\right) \\
& \times \sum_{x_{i-3 / 2} \leq x_{j}+x_{k}<x_{i-1 / 2}}^{j \geq k}\left(1-\frac{1}{2} \delta_{j, k}\right) \beta\left(x_{k}, x_{j}\right) \hat{N}_{j}(t) \hat{N}_{k}(t) \\
& +\left[\lambda_{i}^{+}\left(\bar{v}_{i}\right) H\left(\bar{v}_{i}-x_{i}\right)+\lambda_{i}^{-}\left(\bar{v}_{i}\right) H\left(x_{i}-\bar{v}_{i}\right)\right] \\
& \times \sum_{x_{i-1 / 2} \leq x_{j}+x_{k}<x_{i+1 / 2}}^{j \geq k}\left(1-\frac{1}{2} \delta_{j, k}\right) \beta\left(x_{k}, x_{j}\right) \hat{N}_{j}(t) \hat{N}_{k}(t) \\
& +\lambda_{i}^{+}\left(\bar{v}_{i+1}\right) H\left(x_{i+1}-\bar{v}_{i+1}\right) \\
& \times \sum_{x_{i+1 / 2} \leq x_{j}+x_{k}<x_{i+3 / 2}}^{j \geq k}\left(1-\frac{1}{2} \delta_{j, k}\right) \beta\left(x_{k}, x_{j}\right) \hat{N}_{j}(t) \hat{N}_{k}(t) \\
& -\hat{N}_{i}(t) \sum_{j=1}^{I} \beta\left(x_{i}, x_{j}\right) \hat{N}_{j}(t) .
\end{aligned}
$$

It should be pointed out here that in this work we consider the following discrete norm

$$
\|\mathbf{N}\|=\sum_{i=1}^{I}\left|N_{i}\right| .
$$

The following lemma is required to show the convergence of the scheme to solve (1.2):

Lemma 2.1. Assume that the coagulation kernel and the initial datum satisfy

$$
\beta \in W^{2, \infty}((0, R) \times(0, R)) \text { and } n^{i n} \in W^{2, \infty}(0, R) .
$$

Then there exists a constant $L(T, R)>0$ such that

$$
\|n(t)\|_{W^{2, \infty}(0, R)} \leq L(T, R) .
$$


Proof. In [16], it is shown that there exists a weak solution $n \in L^{\infty}\left((0, T) ; L^{1}(0, R)\right)$ to (1.2) with the suitable initial datum $n^{\text {in }}$. Let $\|\cdot\|_{\infty, 1}$ denotes the norm in $L^{\infty}\left((0, T) ; L^{1}(0, R)\right)$. From $(2.18)$, we can also say that

$$
\beta \in W^{1, \infty}((0, R) \times(0, R)) \text { and } n^{\text {in }} \in W^{1, \infty}(0, R) .
$$

Hence, by Proposition 3.6 in [1], there exists a $C(T, R)>0$ such that

$$
\|n(t)\|_{W^{1, \infty}(0, R)} \leq C(T, R) .
$$

In order to prove (2.19), we use (2.20) and (2.18).

First, we integrate equation (1.2) with respect to $t$ and then differentiate it twice with respect to $x$ to obtain

$$
\begin{aligned}
\frac{\partial^{2} n(t, x)}{\partial x^{2}}= & \frac{\partial^{2} n_{0}(x)}{\partial x^{2}}+\int_{0}^{t}\left[\frac { 1 } { 2 } \int _ { 0 } ^ { x } \left(\frac{\partial^{2} \beta(x-y, y)}{\partial x^{2}} n(x-y, s) n(y, s)\right.\right. \\
& \left.+2 \frac{\partial \beta(x-y, y)}{\partial x} \frac{\partial n(x-y, s)}{\partial x} n(y, s)+\beta(x-y, y) \frac{\partial^{2} n(x-y, s)}{\partial x^{2}} n(y, s)\right) \mathrm{d} y \\
& +\left.\frac{1}{2} \frac{\partial \beta(x-y, y)}{\partial x}\right|_{y=x} n(x, s) n(0, s)-\int_{0}^{R}\left(\frac{\partial^{2} \beta(x, y)}{\partial x^{2}} n(x, s) n(y, s)\right. \\
& \left.\left.+2 \frac{\partial \beta(x, y)}{\partial x} \frac{\partial n(x, s)}{\partial x} n(y, s)+\beta(x, y) \frac{\partial^{2} n(x, s)}{\partial x^{2}} n(y, s)\right) \mathrm{d} y\right] \mathrm{d} s
\end{aligned}
$$

Taking the maximum value over all possible values of $x$, we find

$$
\begin{aligned}
\left\|\frac{\partial^{2} n(t, x)}{\partial x^{2}}\right\|_{L^{\infty}} \leq & \left\|\frac{\partial^{2} n_{0}(x)}{\partial x^{2}}\right\|_{L^{\infty}}+\left\{\frac{3}{2}\|\beta\|_{W^{2, \infty}}\|n\|_{L^{\infty}}\|n\|_{\infty, 1}+\frac{1}{2}\|\beta\|_{W^{1, \infty}}\|n\|_{L^{\infty}}^{2}\right\} t \\
& +3\|\beta\|_{W^{1, \infty}}\|n\|_{\infty, 1} \int_{0}^{t}\left\|\frac{\partial n}{\partial x}\right\|_{L^{\infty}} \mathrm{d} s \\
& +\frac{3}{2}\|\beta\|_{L^{\infty}}\|n\|_{\infty, 1} \int_{0}^{t}\left\|\frac{\partial^{2} n}{\partial x^{2}}\right\|_{L^{\infty}} \mathrm{d} s .
\end{aligned}
$$

Owing to (2.20), we thank Gronwall's lemma which gives us (2.19).

Before moving to the main result, let us recall some definitions and a result taken from [18].

Definition 2.2. The local discretization error is defined by the residual left by substituting the exact solution $\mathbf{N}(t)$ into equation (2.12) as

$$
\sigma(t)=\frac{d \mathbf{N}(t)}{d t}-(\hat{\mathbf{B}}(\mathbf{N}(t))-\hat{\mathbf{D}}(\mathbf{N}(t))) .
$$

The scheme (2.12) is called consistent of order $p$ if, for $\Delta x \rightarrow 0$,

$$
\|\sigma(t)\|=\mathcal{O}\left(\Delta x^{p}\right), \quad \text { uniformly for all } t, 0 \leq t \leq T .
$$

Definition 2.3. The global discretization error is defined by

$$
\epsilon(t)=\mathbf{N}(t)-\hat{\mathbf{N}}(t)
$$

The scheme (2.12) is called convergent of order $p$ if, for $\Delta x \rightarrow 0$,

$$
\|\epsilon(t)\|=\mathcal{O}\left(\Delta x^{p}\right), \quad \text { uniformly for all } t, 0 \leq t \leq T .
$$


It is important that the solution obtained by CAT remains non-negative for all times. This can be easily shown by using the next well known theorem. In the following theorem we write $\hat{\mathbf{M}} \geq 0$ for a vector $\hat{\mathbf{M}} \in \mathbb{R}^{I}$ if all of its components are non-negative.

Theorem 2.4. Suppose that $\hat{\mathbf{F}}(t, \hat{\mathbf{M}})$ defined in (2.12) is continuous and satisfies the Lipschitz condition as

$$
\|\hat{\mathbf{F}}(t, \hat{\mathbf{P}})-\hat{\mathbf{F}}(t, \hat{\mathbf{M}})\| \leq L\|\hat{\mathbf{P}}-\hat{\mathbf{M}}\| \quad \text { for all } \hat{\mathbf{P}}, \hat{\mathbf{M}} \in \mathbb{R}^{I}
$$

Then the solution of the semi-discrete system (2.12) is non-negative if and only if for any vector $\hat{\mathbf{M}} \in \mathbb{R}^{I}$ with $\hat{\mathbf{M}} \geq 0$, the condition $\hat{M}_{i}=0$ implies $\hat{F}_{i}(t, \hat{\mathbf{M}}) \geq 0$ for any $i=1, \ldots, I$ and all $t \geq 0$.

Proof. The proof can be found in ([18], Chap. 1, Thm. 7.1).

The following theorem is required to show the convergence of the CAT.

Theorem 2.5. Let us assume that the Lipschitz conditions on $\hat{\mathbf{B}}(\mathbf{N}(t))$ and $\hat{\mathbf{D}}(\mathbf{N}(t))$ are satisfied for $0 \leq t \leq T$ and for all $\mathbf{N}, \hat{\mathbf{N}} \in \mathbb{R}^{I}$ where $\mathbf{N}$ and $\hat{\mathbf{N}}$ are the projected exact and numerical solutions defined in (2.5) and (2.12) respectively. Then a consistent discretization method is also convergent and the convergence is of the same order as the consistency.

Proof. The proof is similar to Theorem 2.4 in [14].

Now we shall state the main result of the paper.

Theorem 2.6. Under the assumptions of Lemma 2.1, the CAT for solving (1.2) is second order convergent on a uniform and first order convergent on a non-uniform smooth (geometric) grid.

Proof. This result can easily be proved by applying Theorem 2.5. In order to fulfill the requirements of Theorem 2.5, for the convergence of the CAT, it is shown in Section 3 that the scheme is second order consistent on a uniform grid and first order consistent on a non-uniform smooth (geometric) grid. Moreover, in Section 4, the birth $\hat{\mathbf{B}}(\mathbf{N}(t))$ and death $\hat{\mathbf{D}}(\mathbf{N}(t))$ terms satisfy the Lipschitz conditions.

\section{Consistency}

Let us describe the four main sections to study the consistency of the CAT for solving SCE (1.2). First, we evaluate the discretization error of the integrated birth and death terms in Sections 3.1 and 3.2, respectively. Then all error terms are summarized, in Section 3.3, to obtain the local discretization error. Finally, in Section 3.4, the two different types of grids are considered to evaluate the order of consistency of the CAT.

\subsection{Discretization error in the birth term}

The integrated birth term of SCE (1.2) over $i$ th cell is given by

$$
B_{i}=\frac{1}{2} \int_{x_{i-1 / 2}}^{x_{i+1 / 2}} \int_{0}^{x} \beta(x-y, y) n(t, x-y) n(t, y) \mathrm{d} y \mathrm{~d} x .
$$

By changing the order of integration we get

$$
\begin{aligned}
B_{i}= & \frac{1}{2} \sum_{j=1}^{i-1} \int_{x_{j-1 / 2}}^{x_{j+1 / 2}} \int_{x_{i-1 / 2}}^{x_{i+1 / 2}} \beta(x-y, y) n(t, x-y) n(t, y) \mathrm{d} x \mathrm{~d} y \\
& +\frac{1}{2} \int_{x_{i-1 / 2}}^{x_{i+1 / 2}} \int_{y}^{x_{i+1 / 2}} \beta(x-y, y) n(t, x-y) n(t, y) \mathrm{d} x \mathrm{~d} y .
\end{aligned}
$$


Now we apply the midpoint rule to the outer integrals in both terms on the right-hand side and use the relationship $N_{i}=n\left(t, x_{i}\right) \Delta x_{i}+\mathcal{O}\left(\Delta x^{3}\right)$ for the midpoint rule to obtain

$$
\begin{aligned}
B_{i}= & \frac{1}{2} \sum_{j=1}^{i-1} N_{j}(t) \int_{x_{i-1 / 2}}^{x_{i+1 / 2}} \beta\left(x-x_{j}, x_{j}\right) n\left(t, x-x_{j}\right) \mathrm{d} x \\
& +\frac{1}{2} N_{i}(t) \int_{x_{i}}^{x_{i+1 / 2}} \beta\left(x-x_{i}, x_{i}\right) n\left(t, x-x_{i}\right) \mathrm{d} x+\mathcal{O}\left(\Delta x^{3}\right), \\
= & : \tilde{B}_{i}+\mathcal{O}\left(\Delta x^{3}\right) .
\end{aligned}
$$

Let us denote the integral terms in $\tilde{B}_{i}$ by $I_{1}$ and $I_{2}$, respectively, and evaluate them separately.

Integral term $I_{1}$ : We consider the first integral term on the right-hand side in (3.1) and use the substitution $x-x_{j}=x^{\prime}$ to get

$$
I_{1}=\frac{1}{2} \sum_{j=1}^{i-1} N_{j}(t) \int_{x_{i-1 / 2}-x_{j}}^{x_{i+1 / 2}-x_{j}} \beta\left(x^{\prime}, x_{j}\right) n\left(t, x^{\prime}\right) \mathrm{d} x^{\prime} .
$$

We now define $l_{i, j}$ and $\gamma_{i, j}$ to be those indices such that the following hold

$$
x_{i-1 / 2}-x_{j} \in \Lambda_{l_{i, j}} \text { and } \gamma_{i, j}:=\operatorname{sgn}\left[\left(x_{i-1 / 2}-x_{j}\right)-x_{l_{i, j}}\right],
$$

where

$$
\operatorname{sgn}(x):= \begin{cases}1 & \text { if } x>0 \\ 0 & \text { if } x=0 \\ -1 & \text { if } x<0 .\end{cases}
$$

By the definition of the indices $l_{i, j}$ and $\gamma_{i, j}$ in (3.3), equation (3.2) can be rewritten as

$$
\begin{aligned}
I_{1}= & \frac{1}{2} \sum_{j=1}^{i-1} N_{j}(t) \int_{x_{i-1 / 2}-x_{j}}^{x_{l_{i, j}+\frac{1}{2} \gamma_{i, j}}} \beta\left(x^{\prime}, x_{j}\right) n\left(t, x^{\prime}\right) \mathrm{d} x^{\prime} \\
& +\frac{1}{2} \sum_{j=1}^{i-1} N_{j}(t) \sum_{k=l_{i, j}+\frac{1}{2}\left(\gamma_{i, j}+1\right)}^{l_{i+1, j}+\frac{1}{2}\left(\gamma_{i+1, j}-1\right)} \int_{x_{k-1 / 2}}^{x_{k+1 / 2}} \beta\left(x^{\prime}, x_{j}\right) n\left(t, x^{\prime}\right) \mathrm{d} x^{\prime} \\
& +\frac{1}{2} \sum_{j=1}^{i-1} N_{j}(t) \int_{x_{l_{i+1, j}+\frac{1}{2} \gamma_{i+1, j}}^{x_{i+1 / 2}-x_{j}}} \beta\left(x^{\prime}, x_{j}\right) n\left(t, x^{\prime}\right) \mathrm{d} x^{\prime} .
\end{aligned}
$$

Let $p$ be the total number of terms in the following sum

$$
\sum_{k=l_{i, j}+\frac{1}{2}\left(\gamma_{i, j}+1\right)}^{l_{i+1, j}+\frac{1}{2}\left(\gamma_{i+1, j}-1\right)} \int_{x_{k-1 / 2}}^{x_{k+1 / 2}} \beta\left(x^{\prime}, x_{j}\right) n\left(t, x^{\prime}\right) \mathrm{d} x^{\prime} .
$$

In particular, let $p:=\#\left\{n: l_{i, j}+\frac{1}{2}\left(\gamma_{i, j}+1\right) \leq n \leq l_{i+1, j}+\frac{1}{2}\left(\gamma_{i+1, j}-1\right)\right\}$ and set

$$
k_{1}:=l_{i, j}+\frac{1}{2}\left(\gamma_{i, j}+1\right), \quad k_{2}:=k_{1}+1, \ldots, \quad k_{p-1}:=k_{1}+(p-2) .
$$


Next, we shall show that $p$ is finite and can be estimated by a constant which is independent of the grid size. By using the definition of the indices $l_{i, j}$ and $\gamma_{i, j}$ in (3.3), we can estimate

$$
(p-2) \Delta x_{\min } \leq \Delta x_{k_{2}}+\Delta x_{k_{3}}+\ldots+\Delta x_{k_{p-1}} \leq \frac{1}{2}\left(\Delta x_{i}+\Delta x_{i+1}\right) \leq \Delta x
$$

which implies using the assumption of quasi uniformity (2.1) that

$$
(p-2) \leq \frac{\Delta x}{\Delta x_{\min }} \leq K \Rightarrow p \leq K+2 .
$$

This means the above sum has uniformly bounded finite number of terms. So we can apply the midpoint rule to the integral in second term on the right hand side and use $N_{k}(t)=n\left(t, x_{k}\right) \Delta x_{k}+\mathcal{O}\left(\Delta x^{3}\right)$ to get

$$
\begin{aligned}
I_{1}= & \frac{1}{2} \sum_{j=1}^{i-1} N_{j}(t) \int_{x_{i-1 / 2}-x_{j}}^{x_{l_{i, j}+\frac{1}{2} \gamma_{i, j}}} \beta\left(x^{\prime}, x_{j}\right) n\left(t, x^{\prime}\right) \mathrm{d} x^{\prime} \\
& +\frac{1}{2} \sum_{j=1}^{i-1} N_{j}(t) \sum_{x_{i-1 / 2} \leq\left(x_{j}+x_{k}\right)<x_{i+1 / 2}} \beta\left(x_{k}, x_{j}\right) N_{k}(t) \\
& +\frac{1}{2} \sum_{j=1}^{i-1} N_{j}(t) \int_{x_{l_{i+1, j}+\frac{1}{2} \gamma_{i+1, j}}^{x_{i+1 / 2}-x_{j}}} \beta\left(x^{\prime}, x_{j}\right) n\left(t, x^{\prime}\right) \mathrm{d} x^{\prime}+\mathcal{O}\left(\Delta x^{3}\right) .
\end{aligned}
$$

Integral term $I_{2}$ : Let us consider the second integral term in (3.1) and use the substitution $x-x_{i}=x^{\prime}$ to estimate

$$
I_{2}=\frac{1}{2} N_{i}(t) \int_{0}^{x_{i+1 / 2}-x_{i}} \beta\left(x^{\prime}, x_{i}\right) n\left(t, x^{\prime}\right) \mathrm{d} x
$$

Again by the definition of the indices $l_{i, j}$ and $\gamma_{i, j}$ in (3.3) we split the above integral as

$$
\begin{aligned}
I_{2}= & \frac{1}{2} N_{i}(t) \sum_{k=1}^{l_{i+1, i}+\frac{1}{2}\left(\gamma_{i+1, i}-1\right)} \int_{x_{k-1 / 2}}^{x_{k+1 / 2}} \beta\left(x^{\prime}, x_{i}\right) n\left(t, x^{\prime}\right) \mathrm{d} x^{\prime} \\
& +\frac{1}{2} N_{i}(t) \int_{x_{l_{i+1, i}+\frac{1}{2} \gamma_{i+1, i}}}^{x_{i+1 / 2}-x_{i}} \beta\left(x^{\prime}, x_{i}\right) n\left(t, x^{\prime}\right) \mathrm{d} x^{\prime} .
\end{aligned}
$$

By applying the midpoint rule in the first term and using the definition of the indices $l_{i, j}$ and $\gamma_{i, j}$, we get

$$
\begin{aligned}
I_{2}= & \frac{1}{2} N_{i}(t) \sum_{x_{i}+x_{k}<x_{i+1 / 2}} \beta\left(x_{k}, x_{i}\right) N_{k}(t) \\
& +\frac{1}{2} N_{i}(t) \int_{x_{l_{i+1, i}+\frac{1}{2} \gamma_{i+1, i}}}^{x_{i+1 / 2}-x_{i}} \beta\left(x^{\prime}, x_{i}\right) n\left(t, x^{\prime}\right) \mathrm{d} x^{\prime}+\mathcal{O}\left(\Delta x^{3}\right) .
\end{aligned}
$$

By substituting (3.5), (3.6) into (3.1) and using (2.6), we estimate

$$
\begin{aligned}
B_{i}= & \hat{B}_{i}+\frac{1}{2} \sum_{j=1}^{i-1} N_{j}(t) \int_{x_{i-1 / 2}-x_{j}}^{x_{l_{i, j}+\frac{1}{2} \gamma_{i, j}}} \beta\left(x^{\prime}, x_{j}\right) n\left(t, x^{\prime}\right) \mathrm{d} x^{\prime} \\
& +\frac{1}{2} \sum_{j=1}^{i} N_{j}(t) \int_{x_{l_{i+1, j}+\frac{1}{2} \gamma_{i+1, j}}}^{x_{i+1 / 2}-x_{j}} \beta\left(x^{\prime}, x_{j}\right) n\left(t, x^{\prime}\right) \mathrm{d} x^{\prime}+\mathcal{O}\left(\Delta x^{3}\right) .
\end{aligned}
$$


Let us denote the sum of the remaining two integrals on the right hand side in (3.7) by the error $E_{1}$ which will be discussed later.

Now we concentrate to evaluate the integrated term $V_{i}-x_{i} B_{i}$ by using (2.3) and (2.8) as follows

$$
V_{i}-x_{i} B_{i}=\frac{1}{2} \int_{x_{i-1 / 2}}^{x_{i+1 / 2}} \int_{0}^{x}\left(x-x_{i}\right) \beta(x-y, y) n(t, x-y) n(t, y) \mathrm{d} y \mathrm{~d} x .
$$

By changing the order of integration we get

$$
\begin{aligned}
V_{i}-x_{i} B_{i}= & \frac{1}{2} \sum_{j=1}^{i-1} \int_{x_{j-1 / 2}}^{x_{j+1 / 2}} \int_{x_{i-1 / 2}}^{x_{i+1 / 2}}\left(x-x_{i}\right) \beta(x-y, y) n(t, x-y) n(t, y) \mathrm{d} x \mathrm{~d} y \\
& +\frac{1}{2} \int_{x_{i-1 / 2}}^{x_{i+1 / 2}} \int_{y}^{x_{i+1 / 2}}\left(x-x_{i}\right) \beta(x-y, y) n(t, x-y) n(t, y) \mathrm{d} x \mathrm{~d} y .
\end{aligned}
$$

Now applying the midpoint rule to the outer integrals in both the terms on the right hand side and using the relationship $N_{i}=n\left(t, x_{i}\right) \Delta x_{i}+\mathcal{O}\left(\Delta x^{3}\right)$ with $\beta(0, \cdot)=0$, we obtain

$$
\begin{aligned}
V_{i}-x_{i} B_{i}= & \frac{1}{2} \sum_{j=1}^{i-1} N_{j}(t) \int_{x_{i-1 / 2}}^{x_{i+1 / 2}}\left(x-x_{i}\right) \beta\left(x-x_{j}, x_{j}\right) n\left(t, x-x_{j}\right) \mathrm{d} x \\
& +\frac{1}{2} N_{i}(t) \int_{x_{i}}^{x_{i+1 / 2}}\left(x-x_{i}\right) \beta\left(x-x_{i}, x_{i}\right) n\left(t, x-x_{i}\right) \mathrm{d} x+\mathcal{O}\left(\Delta x^{5}\right), \\
= & : \tilde{V}_{i}-x_{i} \tilde{B}_{i}+\mathcal{O}\left(\Delta x^{5}\right) .
\end{aligned}
$$

We denote the integral terms involving in $\tilde{V}_{i}-x_{i} \tilde{B}_{i}$ by $P_{1}$ and $P_{2}$, respectively, and calculate them separately.

Integral term $P_{1}$ : Let us consider the first integral term in (3.8) and insert $x-x_{j}=x^{\prime}$ to estimate

$$
P_{1}=\frac{1}{2} \sum_{j=1}^{i-1} N_{j}(t) \int_{x_{i-1 / 2}-x_{j}}^{x_{i+1 / 2}-x_{j}}\left(x^{\prime}-x_{i}+x_{j}\right) \beta\left(x^{\prime}, x_{j}\right) n\left(t, x^{\prime}\right) \mathrm{d} x^{\prime} .
$$

By the definition of the indices $l_{i, j}$ and $\gamma_{i, j}$ in (3.3), (3.9) can be rewritten as

$$
\begin{aligned}
P_{1}= & \frac{1}{2} \sum_{j=1}^{i-1} N_{j}(t) \int_{x_{i-1 / 2}-x_{j}}^{x_{l_{i, j}+\frac{1}{2} \gamma_{i, j}}}\left(x^{\prime}-x_{i}+x_{j}\right) \beta\left(x^{\prime}, x_{j}\right) n\left(t, x^{\prime}\right) \mathrm{d} x^{\prime} \\
& +\frac{1}{2} \sum_{j=1}^{i-1} N_{j}(t) \sum_{k=l_{i, j}+\frac{1}{2}\left(\gamma_{i, j}+1\right)}^{l_{i+1, j}+\frac{1}{2}\left(\gamma_{i+1, j}-1\right)} \int_{x_{k-1 / 2}}^{x_{k+1 / 2}}\left(x^{\prime}-x_{i}+x_{j}\right) \beta\left(x^{\prime}, x_{j}\right) n\left(t, x^{\prime}\right) \mathrm{d} x^{\prime} \\
& +\frac{1}{2} \sum_{j=1}^{i-1} N_{j}(t) \int_{x_{l_{i+1, j}+\frac{1}{2} \gamma_{i+1, j}}^{x_{i+1 / 2}-x_{j}}}\left(x^{\prime}-x_{i}+x_{j}\right) \beta\left(x^{\prime}, x_{j}\right) n\left(t, x^{\prime}\right) \mathrm{d} x^{\prime} .
\end{aligned}
$$

Since the number of terms in the inner summation of second term on the right hand side is finite as before, therefore we can use the midpoint rule to the integral in second term on the right hand side and use 
$N_{k}(t)=n\left(t, x_{k}\right) \Delta x_{k}+\mathcal{O}\left(\Delta x^{3}\right)$ to obtain

$$
\begin{aligned}
P_{1}= & \frac{1}{2} \sum_{j=1}^{i-1} N_{j}(t) \int_{x_{i-1 / 2}-x_{j}}^{x_{l_{i, j}+\frac{1}{2} \gamma_{i, j}}}\left(x^{\prime}-x_{i}+x_{j}\right) \beta\left(x^{\prime}, x_{j}\right) n\left(t, x^{\prime}\right) \mathrm{d} x^{\prime} \\
& +\frac{1}{2} \sum_{j=1}^{i-1} N_{j}(t) \sum_{x_{i-1 / 2} \leq\left(x_{j}+x_{k}\right)<x_{i+1 / 2}}\left(x_{k}-x_{i}+x_{j}\right) \beta\left(x_{k}, x_{j}\right) N_{k}(t) \\
& +\frac{1}{2} \sum_{j=1}^{i-1} N_{j}(t) \int_{x_{l_{i+1, j}+\frac{1}{2} \gamma_{i+1, j}}^{x_{i+1 / 2}-x_{j}}\left(x^{\prime}-x_{i}+x_{j}\right) \beta\left(x^{\prime}, x_{j}\right) n\left(t, x^{\prime}\right) \mathrm{d} x^{\prime}}^{l_{i+1, j}+\frac{1}{2}\left(\gamma_{i+1, j}-1\right)} \Delta x_{k}^{3} \frac{\partial}{\partial x^{\prime}}\left\{\beta\left(x_{k}, x_{j}\right) n\left(t, x_{k}\right)\right\} \\
& +\frac{1}{24} \sum_{j=1}^{i-1} N_{j}(t) \sum_{k=l_{i, j}+\frac{1}{2}\left(\gamma_{i, j}+1\right)} \\
& +\mathcal{O}\left(\Delta x^{4}\right) .
\end{aligned}
$$

Integral term $P_{2}$ : Let us consider the second integral term in (3.8) and use the substitution $x-x_{i}=x^{\prime}$ to estimate

$$
P_{2}=\frac{1}{2} N_{i}(t) \int_{0}^{x_{i+1 / 2}-x_{i}} x^{\prime} \beta\left(x^{\prime}, x_{i}\right) n\left(t, x^{\prime}\right) \mathrm{d} x .
$$

By the definition of the indices $l_{i, j}$ and $\gamma_{i, j}$ in (3.3) we split the above integral as

$$
\begin{aligned}
P_{2}= & \frac{1}{2} N_{i}(t) \sum_{k=1}^{l_{i+1, i}+\frac{1}{2}\left(\gamma_{i+1, i}-1\right)} \int_{x_{k-1 / 2}}^{x_{k+1 / 2}} x^{\prime} \beta\left(x^{\prime}, x_{i}\right) n\left(t, x^{\prime}\right) \mathrm{d} x^{\prime} \\
& +\frac{1}{2} N_{i}(t) \int_{x_{l_{i+1, i}+\frac{1}{2} \gamma_{i+1, i}}^{x_{i+1 / 2}-x_{i}}}^{x^{\prime} \beta\left(x^{\prime}, x_{i}\right) n\left(t, x^{\prime}\right) \mathrm{d} x^{\prime} .}
\end{aligned}
$$

We apply the midpoint rule in the first term on the right hand side to obtain

$$
\begin{aligned}
P_{2}= & \frac{1}{2} N_{i}(t) \sum_{x_{i}+x_{k}<x_{i+1 / 2}}\left(x_{i}+x_{k}-x_{i}\right) \beta\left(x_{k}, x_{i}\right) N_{k}(t) \\
& +\frac{1}{2} N_{i}(t) \int_{x_{l_{i+1, i}+\frac{1}{2} \gamma_{i+1, i}}}^{x_{i+1 / 2}-x_{i}} x^{\prime} \beta\left(x^{\prime}, x_{i}\right) n\left(t, x^{\prime}\right) \mathrm{d} x^{\prime}+\mathcal{O}\left(\Delta x^{4}\right) .
\end{aligned}
$$

By substituting (3.10), (3.11) into (3.8), and using (2.9) and (2.6), we have

$$
\begin{aligned}
& V_{i}-x_{i} B_{i}=\hat{V}_{i}-x_{i} \hat{B}_{i} \\
& +\frac{1}{2} \sum_{j=1}^{i-1} N_{j}(t) \int_{x_{i-1 / 2}-x_{j}}^{x_{l_{i, j}+\frac{1}{2} \gamma_{i, j}}}\left(x^{\prime}-x_{i}+x_{j}\right) \beta\left(x^{\prime}, x_{j}\right) n\left(t, x^{\prime}\right) \mathrm{d} x^{\prime} \\
& \times \frac{1}{2} \sum_{j=1}^{i} N_{j}(t) \int_{x_{l_{i+1, j}+\frac{1}{2} \gamma_{i+1, j}}^{x_{i+1 / 2}-x_{j}}}\left(x^{\prime}-x_{i}+x_{j}\right) \beta\left(x^{\prime}, x_{j}\right) n\left(t, x^{\prime}\right) \mathrm{d} x^{\prime} \\
& +\frac{1}{24} \sum_{j=1}^{i-1} N_{j}(t) \sum_{k=l_{i, j}+\frac{1}{2}\left(\gamma_{i, j}+1\right)}^{l_{i+1, j}+\frac{1}{2}\left(\gamma_{i+1, j}-1\right)} \Delta x_{k}{ }^{3} \frac{\partial}{\partial x^{\prime}}\left\{\beta\left(x_{k}, x_{j}\right) n\left(t, x_{k}\right)\right\} \\
& +\mathcal{O}\left(\Delta x^{4}\right) \text {. }
\end{aligned}
$$


Now we evaluate the each term in (2.15) separately. We begin with the first term without Heaviside function $H(x)$ and insert the value of $\lambda$ from (2.14) to get

$$
\lambda_{i}^{-}\left(\bar{v}_{i-1}\right) \hat{B}_{i-1}=\frac{\bar{v}_{i-1}-x_{i-1}}{x_{i}-x_{i-1}} \hat{B}_{i-1}=\frac{2}{\Delta x_{i}+\Delta x_{i-1}}\left[\hat{V}_{i-1}-x_{i-1} \hat{B}_{i-1}\right] .
$$

Using the equation (3.8) and (3.12), we obtain

$$
\begin{aligned}
\lambda_{i}^{-}\left(\bar{v}_{i-1}\right) \hat{B}_{i-1}= & \frac{2}{\Delta x_{i}+\Delta x_{i-1}}\left[\tilde{V}_{i-1}-x_{i-1} \tilde{B}_{i-1}\right. \\
& -\frac{1}{2} \sum_{j=1}^{i-2} N_{j}(t) \int_{x_{i-3 / 2}-x_{j}}^{x_{l_{i-1, j}+\frac{1}{2} \gamma_{i-1, j}}}\left(x^{\prime}-x_{i-1}+x_{j}\right) \beta\left(x^{\prime}, x_{j}\right) n\left(t, x^{\prime}\right) \mathrm{d} x^{\prime} \\
& -\frac{1}{2} \sum_{j=1}^{i-1} N_{j}(t) \int_{x_{l_{i, j}+\frac{1}{2} \gamma_{i, j}}^{x_{i-1 / 2}-x_{j}}\left(x^{\prime}-x_{i-1}+x_{j}\right) \beta\left(x^{\prime}, x_{j}\right) n\left(t, x^{\prime}\right) \mathrm{d} x^{\prime}}^{l_{i, j}+\frac{1}{2}\left(\gamma_{i, j}-1\right)} \Delta x_{k}^{3} \frac{\partial}{\partial x^{\prime}}\left\{\beta\left(x_{k}, x_{j}\right) n\left(t, x_{k}\right)\right\} \\
& -\frac{1}{24} \sum_{j=1}^{i-2} N_{j}(t) \sum_{k=l_{i-1, j}+\frac{1}{2}\left(\gamma_{i-1, j}+1\right)} \\
& \left.+\mathcal{O}\left(\Delta x^{4}\right)\right] .
\end{aligned}
$$

In order to solve equation (3.13), we estimate $\tilde{V}_{i-1}-x_{i-1} \tilde{B}_{i-1}$ defined in (3.8) as follows

$$
\begin{aligned}
\tilde{V}_{i-1}-x_{i-1} \tilde{B}_{i-1}= & \frac{1}{2}\left[\sum_{j=1}^{i-2} N_{j} \int_{x_{i-3 / 2}}^{x_{i-1 / 2}}\left(x-x_{i-1}\right) f\left(x-x_{j}, x_{j}\right) \mathrm{d} x\right. \\
& \left.+\int_{x_{i-1}}^{x_{i-1 / 2}}\left(x-x_{i-1}\right) f\left(x-x_{i-1}, x_{i-1}\right) n\left(t, x_{i-1}\right) \Delta x_{i-1} \mathrm{~d} x\right],
\end{aligned}
$$

where $f(\cdot, y):=\beta(\cdot, y) n(t, \cdot)$. Next, we use Taylor series expansions of each integrand about $x_{i-1}$ in equation $(3.14)$ as

$$
\begin{aligned}
\left(x-x_{i-1}\right) f\left(x-x_{j}, x_{j}\right)= & 0+f\left(x_{i-1}-x_{j}, x_{j}\right)\left(x-x_{i-1}\right) \\
& +f_{x}\left(x_{i-1}-x_{j}, x_{j}\right)\left(x-x_{i-1}\right)^{2}+\mathcal{O}\left(\Delta x^{3}\right), \\
\left(x-x_{i-1}\right) f\left(x-x_{i-1}, x_{i-1}\right)= & 0+f\left(x_{i-1}-x_{i-1}, x_{i-1}\right)\left(x-x_{i-1}\right)+\mathcal{O}\left(\Delta x^{2}\right) .
\end{aligned}
$$

The substitution of the above Taylor series expansion in equation (3.14) gives

$$
\tilde{V}_{i-1}-x_{i-1} \tilde{B}_{i-1}=\frac{1}{2}\left[\frac{1}{12} \sum_{j=1}^{i-2} N_{j} f_{x}\left(x_{i-1}-x_{j}, x_{j}\right) \Delta x_{i-1}^{3}+\frac{1}{8} f\left(x_{i-1}-x_{i-1}, x_{i-1}\right) n\left(t, x_{i-1}\right) \Delta x_{i-1}^{3}+\mathcal{O}\left(\Delta x^{4}\right)\right] \text {. }
$$

Since $\beta\left(x_{i-1}-x_{i-1}, x_{i-1}\right)=\beta\left(0, x_{i-1}\right)=0$, therefore we have $f\left(x_{i-1}-x_{i-1}, x_{i-1}\right)=0$. This implies that

$$
\tilde{V}_{i-1}-x_{i-1} \tilde{B}_{i-1}=\frac{1}{24} \sum_{j=1}^{i-2} N_{j} f_{x}\left(x_{i-1}-x_{j}, x_{j}\right) \Delta x_{i-1}^{3}+\mathcal{O}\left(\Delta x^{4}\right) .
$$

Again the application of Taylor series expansion gives us

$$
\tilde{V}_{i-1}-x_{i-1} \tilde{B}_{i-1}=\frac{1}{24} \sum_{j=1}^{i-2} N_{j} f_{x}\left(x_{i}-x_{j}, x_{j}\right) \Delta x_{i-1}^{3}+\mathcal{O}\left(\Delta x^{4}\right) .
$$


Finally, substituting (3.15) into (3.13), we obtain

$$
\begin{aligned}
\lambda_{i}^{-}\left(\bar{v}_{i-1}\right) \hat{B}_{i-1}= & \frac{1}{12} \sum_{j=1}^{i-2} N_{j} f_{x}\left(x_{i}-x_{j}, x_{j}\right) \frac{\Delta x_{i-1}^{3}}{\Delta x_{i}+\Delta x_{i-1}} \\
& -\sum_{j=1}^{i-2} N_{j}(t) \int_{x_{i-3 / 2}-x_{j}}^{x_{l_{i-1, j}+\frac{1}{2} \gamma_{i-1, j}}} \frac{\left(x^{\prime}-x_{i-1}+x_{j}\right)}{\Delta x_{i}+\Delta x_{i-1}} f\left(x^{\prime}, x_{j}\right) \mathrm{d} x^{\prime} \\
& -\sum_{j=1}^{i-1} N_{j}(t) \int_{x_{l_{i, j}+\frac{1}{2} \gamma_{i, j}}^{x_{i-1 / 2}-x_{j}} \frac{\left(x^{\prime}-x_{i-1}+x_{j}\right)}{\Delta x_{i}+\Delta x_{i-1}} f\left(x^{\prime}, x_{j}\right) \mathrm{d} x^{\prime}}^{l_{i, j}+\frac{1}{2}\left(\gamma_{i, j}-1\right)} \frac{\Delta x_{k}{ }^{3}}{\Delta x_{i}+\Delta x_{i-1}} f_{x^{\prime}}\left(x_{k}, x_{j}\right) \\
& -\frac{1}{12} \sum_{j=1}^{i-2} N_{j}(t) \sum_{k=l_{i-1, j}+\frac{1}{2}\left(\gamma_{i-1, j}+1\right)} \\
& +\mathcal{O}\left(\Delta x^{3}\right) .
\end{aligned}
$$

Next, the second term in (2.15) is evaluated as

$$
\begin{aligned}
\lambda_{i}^{+}\left(\bar{v}_{i}\right) \hat{B}_{i} & =\frac{\bar{v}_{i}-x_{i+1}}{x_{i}-x_{i+1}} \hat{B}_{i}=\left(1-\frac{\bar{v}_{i}-x_{i}}{x_{i+1}-x_{i}}\right) \hat{B}_{i} \\
& =\hat{B}_{i}-\frac{2}{\Delta x_{i+1}+\Delta x_{i}}\left(\hat{V}_{i}-x_{i} \hat{B}_{i}\right) .
\end{aligned}
$$

Calculating as before, we estimate the above expression in the following form

$$
\begin{aligned}
\lambda_{i}^{+}\left(\bar{v}_{i}\right) \hat{B}_{i}= & \hat{B}_{i}-\frac{1}{12} \sum_{j=1}^{i-1} N_{j} f_{x}\left(x_{i}-x_{j}, x_{j}\right) \frac{\Delta x_{i}^{3}}{\Delta x_{i}+\Delta x_{i+1}} \\
& +\sum_{j=1}^{i-1} N_{j}(t) \int_{x_{i-1 / 2}-x_{j}}^{x_{l_{i, j}+\frac{1}{2} \gamma_{i, j}}} \frac{\left(x^{\prime}-x_{i}+x_{j}\right)}{\Delta x_{i}+\Delta x_{i+1}} f\left(x^{\prime}, x_{j}\right) \mathrm{d} x^{\prime} \\
& +\sum_{j=1}^{i} N_{j}(t) \int_{x_{l_{i+1, j}+\frac{1}{2} \gamma_{i+1, j}}^{x_{i+1 / 2}-x_{j}} \frac{\left(x^{\prime}-x_{i}+x_{j}\right)}{\Delta x_{i}+\Delta x_{i+1}} f\left(x^{\prime}, x_{j}\right) \mathrm{d} x^{\prime}}^{l_{i+1, j}+\frac{1}{2}\left(\gamma_{i+1, j}-1\right)} \frac{\Delta x_{k}{ }^{3}}{\Delta x_{i}+\Delta x_{i+1}} f_{x^{\prime}}\left(x_{k}, x_{j}\right)+\mathcal{O}\left(\Delta x^{3}\right) .
\end{aligned}
$$

Similar to the second term we obtain

$$
\begin{aligned}
& \lambda_{i}^{-}\left(\bar{v}_{i}\right) \hat{B}_{i}=\hat{B}_{i}+\frac{1}{12} \sum_{j=1}^{i-1} N_{j} f_{x}\left(x_{i}-x_{j}, x_{j}\right) \frac{\Delta x_{i}^{3}}{\Delta x_{i}+\Delta x_{i-1}} \\
& -\sum_{j=1}^{i-1} N_{j}(t) \int_{x_{i-1 / 2}-x_{j}}^{x_{l_{i, j}+\frac{1}{2} \gamma_{i, j}}} \frac{\left(x^{\prime}-x_{i}+x_{j}\right)}{\Delta x_{i}+\Delta x_{i-1}} f\left(x^{\prime}, x_{j}\right) \mathrm{d} x^{\prime} \\
& -\sum_{j=1}^{i} N_{j}(t) \int_{x_{l_{i+1, j}+\frac{1}{2} \gamma_{i+1, j}}^{x_{i+1 / 2}-x_{j}}} \frac{\left(x^{\prime}-x_{i}+x_{j}\right)}{\Delta x_{i}+\Delta x_{i-1}} f\left(x^{\prime}, x_{j}\right) \mathrm{d} x^{\prime} \\
& -\frac{1}{12} \sum_{j=1}^{i-1} N_{j}(t) \sum_{k=l_{i, j}+\frac{1}{2}\left(\gamma_{i, j}+1\right)}^{l_{i+1, j}+\frac{1}{2}\left(\gamma_{i+1, j}-1\right)} \frac{\Delta x_{k}{ }^{3}}{\Delta x_{i}+\Delta x_{i-1}} f_{x^{\prime}}\left(x_{k}, x_{j}\right)+\mathcal{O}\left(\Delta x^{3}\right) .
\end{aligned}
$$


Finally, similar to the first term we can easily estimate

$$
\begin{aligned}
\lambda_{i}^{+}\left(\bar{v}_{i+1}\right) \hat{B}_{i+1}= & \frac{1}{12} \sum_{j=1}^{i} N_{j} f_{x}\left(x_{i}-x_{j}, x_{j}\right) \frac{\Delta x_{i+1}^{3}}{\Delta x_{i}+\Delta x_{i+1}} \\
& +\sum_{j=1}^{i} N_{j}(t) \int_{x_{i+1 / 2}-x_{j}}^{x_{l_{i+1, j}+\frac{1}{2} \gamma_{i+1, j}}} \frac{\left(x^{\prime}-x_{i+1}+x_{j}\right)}{\Delta x_{i}+\Delta x_{i+1}} f\left(x^{\prime}, x_{j}\right) \mathrm{d} x^{\prime} \\
& +\sum_{j=1}^{i+1} N_{j}(t) \int_{x_{l_{i+2, j}+\frac{1}{2} \gamma_{i+2, j}}^{x_{i+3 / 2}-x_{j}}}^{\frac{\left(x^{\prime}-x_{i+1}+x_{j}\right)}{\Delta x_{i}+\Delta x_{i+1}} f\left(x^{\prime}, x_{j}\right) \mathrm{d} x^{\prime}} \\
& +\frac{1}{12} \sum_{j=1}^{i} N_{j}(t) \sum_{k=l_{i+1, j}+\frac{1}{2}\left(\gamma_{i+1, j}+1\right)}^{l_{i+2, j}\left(\gamma_{i+2, j}-1\right)} \frac{\Delta x_{k}^{3}}{\Delta x_{i}+\Delta x_{i+1}} f_{x^{\prime}}\left(x_{k}, x_{j}\right)+\mathcal{O}\left(\Delta x^{3}\right) .
\end{aligned}
$$

By substituting (3.16)-(3.19) into (2.15) and using (3.7), the local discretization error can be evaluated as follows:

Case I: $\bar{v}_{i-1}>x_{i-1}, \bar{v}_{i}>x_{i}$ and $\bar{v}_{i+1} \geq x_{i+1}$ :

$$
\begin{aligned}
& \hat{B}_{i}^{C A}=B_{i} \\
& \left.\begin{array}{c}
-\frac{1}{2} \sum_{j=1}^{i-1} N_{j}(t) \int_{x_{i-1 / 2}-x_{j}}^{x_{l_{i, j}+\frac{1}{2} \gamma_{i, j}}} f\left(x^{\prime}, x_{j}\right) \mathrm{d} x^{\prime} \\
-\frac{1}{2} \sum_{j=1}^{i} N_{j}(t) \int_{x_{l_{i+1, j}+\frac{1}{2} \gamma_{i+1, j}}}^{x_{i+1 / 2}-x_{j}} f\left(x^{\prime}, x_{j}\right) \mathrm{d} x^{\prime}
\end{array}\right\}=: E_{1} \\
& \left.+\frac{1}{12}\left(\frac{\Delta x_{i-1}{ }^{3}}{\Delta x_{i}+\Delta x_{i-1}}-\frac{\Delta x_{i}{ }^{3}}{\Delta x_{i}+\Delta x_{i+1}}\right) \sum_{j=1}^{i} N_{j} f_{x^{\prime}}\left(x_{i}-x_{j}, x_{j}\right)\right\}=: E_{2} \\
& +\sum_{j=1}^{i-1} N_{j}(t) \int_{x_{i-1 / 2}-x_{j}}^{x_{l_{i, j}+\frac{1}{2} \gamma_{i, j}}} \frac{\left(x^{\prime}-x_{i}+x_{j}\right)}{\Delta x_{i}+\Delta x_{i+1}} f\left(x^{\prime}, x_{j}\right) \mathrm{d} x^{\prime} \\
& +\sum_{j=1}^{i} N_{j}(t) \int_{x_{l_{i+1, j}+\frac{1}{2} \gamma_{i+1, j}}^{x_{i+1 / 2}-x_{j}}} \frac{\left(x^{\prime}-x_{i}+x_{j}\right)}{\Delta x_{i}+\Delta x_{i+1}} f\left(x^{\prime}, x_{j}\right) \mathrm{d} x^{\prime} \\
& -\sum_{j=1}^{i-2} N_{j}(t) \int_{x_{i-3 / 2}-x_{j}}^{x_{l_{i-1, j}+\frac{1}{2} \gamma_{i-1, j}}} \frac{\left(x^{\prime}-x_{i-1}+x_{j}\right)}{\Delta x_{i}+\Delta x_{i-1}} f\left(x^{\prime}, x_{j}\right) \mathrm{d} x^{\prime} \\
& \left.-\sum_{j=1}^{i-1} N_{j}(t) \int_{x_{l_{i, j}+\frac{1}{2} \gamma_{i, j}}^{x_{i-1 / 2}-x_{j}}} \frac{\left(x^{\prime}-x_{i-1}+x_{j}\right)}{\Delta x_{i}+\Delta x_{i-1}} f\left(x^{\prime}, x_{j}\right) \mathrm{d} x^{\prime}\right) \\
& +\frac{1}{12} \sum_{j=1}^{i-1} N_{j}(t) \sum_{k=l_{i, j}+\frac{1}{2}\left(\gamma_{i, j}+1\right)}^{l_{i+1, j}+\frac{1}{2}\left(\gamma_{i+1, j}-1\right)} \frac{\Delta x_{k}{ }^{3}}{\Delta x_{i}+\Delta x_{i+1}} f_{x^{\prime}}\left(x_{k}, x_{j}\right) \\
& \left.-\frac{1}{12} \sum_{j=1}^{i-2} N_{j}(t) \sum_{k=l_{i-1, j}+\frac{1}{2}\left(\gamma_{i-1, j}+1\right)}^{\substack{k=l_{i, j}+\frac{1}{2}\left(\gamma_{i, j}+1\right) \\
l_{i, j}+\frac{1}{2}\left(\gamma_{i, j}-1\right)}} \frac{\Delta x_{k}{ }^{3}}{\Delta x_{i}+\Delta x_{i-1}} f_{x^{\prime}}\left(x_{k}, x_{j}\right)\right\} \\
& +\mathcal{O}\left(\Delta x^{3}\right),
\end{aligned}
$$

where $E_{1}, E_{2}, E_{3}$, and $E_{4}$ are the error terms. 
Case II: $\bar{v}_{i-1} \leq x_{i-1}, \bar{v}_{i}<x_{i}$ and $\bar{v}_{i+1}<x_{i+1}$ : Similar to the previous case, we have

$$
\hat{B}_{i}^{C A}=B_{i}+E_{1}+E_{2}^{\prime}+E_{3}^{\prime}+E_{4}^{\prime}+\mathcal{O}\left(\Delta x^{3}\right),
$$

where the error $E_{2}^{\prime}$ will have the same expression as $E_{2}$, defined in (3.20), except only two terms $\Delta x_{i-1}{ }^{3}$ and $\Delta x_{i}{ }^{3}$ which are, respectively, replaced by $\Delta x_{i}{ }^{3}$ and $\Delta x_{i+1}{ }^{3}$. The expressions for $E_{3}^{\prime}$ and $E_{4}^{\prime}$ can easily be written as $E_{3}$ and $E_{4}$, respectively, just with the replacement of $i$ by $i+1$ except in the denominators involved in $E_{3}$ and $E_{4}$.

Case III: $\bar{v}_{i-1} \leq x_{i-1}, \bar{v}_{i}=x_{i}$ and $\bar{v}_{i+1} \geq x_{i+1}$

$$
\hat{B}_{i}^{C A}=B_{i}+E_{1}+\mathcal{O}\left(\Delta x^{3}\right) .
$$

\subsection{Discretization error in the death term}

Next, the discretization error for death term is calculated in the $i$ th cell. From equation (2.4), the integrated death term can be written as follows

$$
D_{i}=\int_{x_{i-1 / 2}}^{x_{i+1 / 2}} \sum_{j=1}^{I} \int_{x_{j-1 / 2}}^{x_{j+1 / 2}} K(x, y) n(t, y) n(t, x) \mathrm{d} y \mathrm{~d} x .
$$

The application of the midpoint rule to the outer and inner integrals gives us

$$
D_{i}=N_{i}(t) \sum_{j=1}^{I} K\left(x_{i}, x_{j}\right) N_{j}(t)+\mathcal{O}\left(\Delta x^{3}\right)=\hat{D}_{i}+\mathcal{O}\left(\Delta x^{3}\right) .
$$

\subsection{Summary of all terms}

From equations (3.20)-(3.22) and (3.23), we can estimate the local discretization error $\sigma_{i}(t)=\left(B_{i}-D_{i}\right)-$ $\left(\hat{B}_{i}^{C A}-\hat{B}_{i}^{C A}\right)$ as

$$
\sigma_{i}(t)= \begin{cases}E_{1}+E_{2}+E_{3}+E_{4}+\mathcal{O}\left(\Delta x^{3}\right) & \text { if } i \in \mathfrak{A}_{1}, \\ E_{1}+E_{2}^{\prime}+E_{3}^{\prime}+E_{4}^{\prime}+\mathcal{O}\left(\Delta x^{3}\right) & \text { if } i \in \mathfrak{A}_{2}, \\ E_{1}+\mathcal{O}\left(\Delta x^{3}\right) & \text { if } i \in \mathfrak{A}_{3} .\end{cases}
$$

where

$$
\begin{aligned}
& \mathfrak{A}_{1}=\left\{i \in \mathbb{N} \mid \bar{v}_{i-1}>x_{i-1}, \bar{v}_{i}>x_{i}, \bar{v}_{i+1} \geq x_{i+1}\right\}, \\
& \mathfrak{A}_{2}=\left\{i \in \mathbb{N} \mid \bar{v}_{i-1} \leq x_{i-1}, \bar{v}_{i}<x_{i}, \bar{v}_{i+1}<x_{i+1}\right\}, \\
& \mathfrak{A}_{3}=\left\{i \in \mathbb{N} \mid \bar{v}_{i-1} \leq x_{i-1}, \bar{v}_{i}=x_{i}, \bar{v}_{i+1} \geq x_{i+1}\right\}
\end{aligned}
$$

Here we consider three different cases to find the order of consistency. Then, the order of consistency is given by

$$
\|\sigma(t)\|=\sum_{i \in \mathfrak{A}_{1}}\left|\sigma_{i}(t)\right|+\sum_{i \in \mathfrak{A}_{2}}\left|\sigma_{i}(t)\right|+\sum_{i \in \mathfrak{A}_{3}}\left|\sigma_{i}(t)\right|
$$

\subsection{Grids}

The following two different types of grids will be considered to find the order of consistency of CAT. 


\subsubsection{Uniform grids}

Let us begin with the case of uniform grids i.e. $\Delta x_{i}=\Delta x$ and $x_{i}=(i-1 / 2) \Delta x$ for any $i=1, \ldots, I$. Here, $E_{2}$ and $E_{2}^{\prime}$ defined in (3.20) and (3.21), respectively, are obviously zero. In case of such uniform grids, we have

$$
x_{i-1 / 2}-x_{j}=x_{i-j}, \quad x_{i+1 / 2}-x_{j}=x_{i-j+1}, \quad \text { and } x_{i-3 / 2}-x_{j}=x_{i-j-1} .
$$

By using the definition of indices $l_{i, j}$ and $\gamma_{i, j}$ in (3.3), we calculate

$$
x_{i-1 / 2}-x_{j}=x_{i-j} \in \Lambda_{l_{i, j}},
$$

which gives

$$
x_{i-1 / 2}-x_{j}=x_{i-j}=x_{l_{i, j}} .
$$

Similarly, we obtain

$$
x_{i+1 / 2}-x_{j}=x_{i-j+1}=x_{l_{i+1, j}},
$$

and

$$
x_{i-3 / 2}-x_{j}=x_{i-j-1}=x_{l_{i-1, j}} .
$$

This shows that $\gamma_{i-1, j}=\gamma_{i, j}=\gamma_{i+1, j}=0$. Therefore, in (3.20)-(3.21), the error terms $E_{1}, E_{3}$ and $E_{3}^{\prime}$ become zero. It can also be easily realized that $x_{i-3 / 2}-x_{j}, x_{i-1 / 2}-x_{j}$, and $x_{i+1 / 2}-x_{j}$ are the pivot points of the adjacent cells, i.e. $l_{i-1, j}=(i-j-1)$ th,$l_{i, j}=(i-j)$ th and $l_{i+1, j}=(i-j+1)$ th cells, respectively. Thus, by substituting the values of all these indices in $E_{4}$ and $E_{4}^{\prime}$ defined in (3.20) and (3.21), respectively, and applying the Taylor series expansion, we obtain $E_{4}=\mathcal{O}\left(\Delta x^{3}\right)$ and $E_{4}^{\prime}=\mathcal{O}\left(\Delta x^{3}\right)$. Then, from (3.24), we obtain

$$
\sigma_{i}(t)=\mathcal{O}\left(\Delta x^{3}\right) \text { if } i \in \mathfrak{A}_{1}, \mathfrak{A}_{2}, \mathfrak{A}_{3} .
$$

By using (3.25), the order of consistency is thus given by

$$
\|\sigma(t)\|=\mathcal{O}\left(\Delta x^{2}\right) .
$$

Therefore, the cell average technique is second order consistent on uniform grids.

\subsubsection{Non-uniform smooth grids}

Non-uniform smooth grids can be obtained by applying some smooth transformation to uniform grids. Assume a variable $\xi$ with uniform grids and a smooth transformation $x=g(\xi)$ such that $x_{i \pm \frac{1}{2}}=g\left(\xi_{i \pm \frac{1}{2}}\right)$ for any $i=1, \ldots, I$ to get non-uniform smooth grids. In this case, we show that the scheme is first order consistent. Let $h$ be the uniform mesh width in the variable $\xi$. For such type of smooth grids, Taylor series expansions in smooth transformations give

$$
\Delta x_{i}=x_{i+\frac{1}{2}}-x_{i-\frac{1}{2}}=g\left(\xi_{i}+\frac{h}{2}\right)-g\left(\xi_{i}-\frac{h}{2}\right)=h g^{\prime}\left(\xi_{i}\right)+\mathcal{O}\left(h^{3}\right) .
$$

Hence, by calculating $\Delta x_{i-1}$ and $\Delta x_{i+1}$ similar to $\Delta x_{i}$, we obtain

$$
\begin{aligned}
& \Delta x_{i}-\Delta x_{i-1}=\mathcal{O}\left(h^{2}\right), \\
& \Delta x_{i}+\Delta x_{i+1}=h\left[g^{\prime}\left(\xi_{i}\right)+g^{\prime}\left(\xi_{i+1}\right)\right]+\mathcal{O}\left(h^{3}\right)=2 h g^{\prime}\left(\xi_{i}\right)+\mathcal{O}\left(h^{2}\right),
\end{aligned}
$$

and similarly, we have

$$
\Delta x_{i}+\Delta x_{i-1}=h\left[g^{\prime}\left(\xi_{i}\right)+g^{\prime}\left(\xi_{i-1}\right)\right]+\mathcal{O}\left(h^{3}\right)=2 h g^{\prime}\left(\xi_{i}\right)+\mathcal{O}\left(h^{2}\right) .
$$

In particular, we deal with a special type of non-uniform smooth grids which is known as geometric grids. Such type of grids can be defined as $x_{i+\frac{1}{2}}=r x_{i-\frac{1}{2}}, r>1, i=1, \ldots, I$. An exponential function can be applied 
on uniform grids as a smooth transformation to construct such type of geometric grids. Mathematically, we write

$$
\begin{aligned}
x_{i+1 / 2}=\exp \left(\xi_{i+1 / 2}\right) & =\exp \left(h+\xi_{i-1 / 2}\right) \\
& =\exp (h) \exp \left(\xi_{i-1 / 2}\right) \\
& =\exp (h) x_{i-1 / 2}=: r x_{i-1 / 2}, r>1 .
\end{aligned}
$$

To solve the error terms appearing in (3.24), let us further assume that $\xi_{11}, \xi_{12}, \xi_{21}, \xi_{22}, \xi_{31}$ and $\xi_{32}$ are corresponding points on uniform mesh for $x_{l_{i+1, j+1}+\frac{1}{2} \gamma_{i+1, j+1}}, x_{i+1 / 2}-x_{j+1}, x_{l_{i, j}+\frac{1}{2} \gamma_{i, j}}, x_{i-1 / 2}-x_{j}, x_{l_{i-1, j-1}+\frac{1}{2} \gamma_{i-1, j-1}}$ and $x_{i-3 / 2}-x_{j-1}$, respectively. Due to an application of exponential smooth transformation, these points can be defined as

$$
\xi_{11}=\ln \left(x_{l_{i+1, j+1}+\frac{1}{2} \gamma_{i+1, j+1}}\right), \ldots, \xi_{32}=\ln \left(x_{i-3 / 2}-x_{j-1}\right) .
$$

By the definition of the indices in (3.3), we know

$$
x_{i+1 / 2}-x_{j+1} \in \Lambda_{l_{i+1, j+1}}, \quad x_{i-1 / 2}-x_{j} \in \Lambda_{l_{i, j}} \text { and } x_{i-3 / 2}-x_{j-1} \in \Lambda_{l_{i-1, j-1}} .
$$

For geometric grids, we have

$$
x_{i+1 / 2}-x_{j+1}=r\left(x_{i-1 / 2}-x_{j}\right)=r^{2}\left(x_{i-3 / 2}-x_{j-1}\right) .
$$

Therefore, we have

$$
l_{i+1, j+1}=l_{i, j}+1=l_{i-1, j-1}+2 .
$$

Further, in case of geometric grids, we have

$$
\gamma_{i+1, j+1}=\gamma_{i, j}=\gamma_{i-1, j-1}
$$

Let us consider

$$
\begin{aligned}
h_{1}=\xi_{11}-\xi_{12}= & \ln \left(x_{l_{i+1, j+1}+\frac{1}{2} \gamma_{i+1, j+1}}\right)-\ln \left(x_{i+1 / 2}-x_{j+1}\right) \\
& =\ln \left(\frac{x_{l_{i+1, j+1}+\frac{1}{2} \gamma_{i+1, j+1}}}{x_{i+1 / 2}-x_{j+1}}\right)=\ln \left(\frac{x_{l_{i, j}+\frac{1}{2} \gamma_{i, j}}}{x_{i-1 / 2}-x_{j}}\right)=\xi_{21}-\xi_{22} \\
& =\ln \left(\frac{x_{l_{i-1, j-1}+\frac{1}{2} \gamma_{i-1, j-1}}}{x_{i-3 / 2}-x_{j-1}}\right)=\xi_{31}-\xi_{32} .
\end{aligned}
$$

Similarly, we estimate

$$
\xi_{12}-\xi_{22}=\ln \left(\frac{x_{i+1 / 2}-x_{j+1}}{x_{i-1 / 2}-x_{j}}\right)=\ln (r)=h,
$$

and

$$
\xi_{22}-\xi_{32}=\ln \left(\frac{x_{i-1 / 2}-x_{j}}{x_{i-3 / 2}-x_{j-1}}\right)=\ln (r)=h .
$$

Again, by application of smooth transformation, we can easily obtain

$$
\begin{aligned}
x_{l_{i+1, j+1}+\frac{1}{2} \gamma_{i+1, j+1}}-\left(x_{i+1 / 2}-x_{j+1}\right) & =g\left(\xi_{11}\right)-g\left(\xi_{12}\right)=h_{1} g^{\prime}\left(\xi_{12}\right)+\mathcal{O}\left(h^{2}\right), \\
x_{l_{i, j}+\frac{1}{2} \gamma_{i, j}}-\left(x_{i-1 / 2}-x_{j}\right)=g\left(\xi_{21}\right)-g\left(\xi_{22}\right) & =h_{1} g^{\prime}\left(\xi_{22}\right)+\mathcal{O}\left(h^{2}\right),
\end{aligned}
$$


and

$$
x_{l_{i-1, j-1}+\frac{1}{2} \gamma_{i-1, j-1}}-\left(x_{i-3 / 2}-x_{j-1}\right)=g\left(\xi_{31}\right)-g\left(\xi_{32}\right)=h_{1} g^{\prime}\left(\xi_{32}\right)+\mathcal{O}\left(h^{2}\right) .
$$

All these identities will play an important role to solve the error terms involved in (3.24), which helps us to calculate the order of local discretization error $\sigma_{i}$.

We first evaluate $E_{1}$ defined in (3.20) as follows

$$
E_{1}=\frac{1}{2} \sum_{j=1}^{i-1} N_{j}(t) \int_{x_{i-1 / 2}-x_{j}}^{x_{l_{i, j}+\frac{1}{2} \gamma_{i, j}}} f\left(x, x_{j}\right) \mathrm{d} x+\frac{1}{2} \sum_{j=1}^{i} N_{j}(t) \int_{x_{l_{i+1, j}+\frac{1}{2} \gamma_{i+1, j}}}^{x_{i+1 / 2}-x_{j}} f\left(x, x_{j}\right) \mathrm{d} x .
$$

Applying the left and right rectangle rules in the integrals involved in the first and second terms, respectively, on the right-hand side, we estimate

$$
\begin{aligned}
E_{1}= & \frac{1}{2} \sum_{j=1}^{i-1} N_{j}(t) f\left(x_{i-1 / 2}-x_{j}, x_{j}\right)\left(x_{l_{i, j}+\frac{1}{2} \gamma_{i, j}}-x_{i-1 / 2}+x_{j}\right) \\
& +\frac{1}{2} \sum_{j=1}^{i} N_{j}(t) f\left(x_{i+1 / 2}-x_{j}, x_{j}\right)\left(x_{i+1 / 2}-x_{j}-x_{l_{i+1, j}+\frac{1}{2} \gamma_{i+1, j}}\right)+\mathcal{O}\left(\Delta x^{2}\right) .
\end{aligned}
$$

Then an application of Taylor's series expansion about $x_{i-1 / 2}=x_{i+1 / 2}$ in $f\left(x_{i-1 / 2}-x_{j}\right)$ gives

$$
\begin{aligned}
E_{1}= & \frac{1}{2} \sum_{j=1}^{i-1} N_{j}(t) f\left(x_{i+1 / 2}-x_{j}, x_{j}\right)\left(x_{l_{i, j}+\frac{1}{2} \gamma_{i, j}}-x_{i-1 / 2}+x_{j}\right) \\
& -\frac{1}{2} \sum_{j=1}^{i} N_{j}(t) f\left(x_{i+1 / 2}-x_{j}, x_{j}\right)\left(x_{l_{i+1, j}+\frac{1}{2} \gamma_{i+1, j}}-x_{i+1 / 2}+x_{j}\right)+\mathcal{O}\left(\Delta x^{2}\right) .
\end{aligned}
$$

We replace $j$ by $j+1$ in the second term on the right-hand side and use the relationship $N_{j}(t)=n\left(t, x_{j}\right) \Delta x_{j}+$ $\mathcal{O}\left(\Delta x^{3}\right)$ for the midpoint rule. Also, we drop the term which is of second order, and obtain

$$
\begin{aligned}
E_{1}= & \frac{1}{2} \sum_{j=1}^{i-1} n\left(t, x_{j}\right) \Delta x_{j} f\left(x_{i+1 / 2}-x_{j}, x_{j}\right)\left(x_{l_{i, j}+\frac{1}{2} \gamma_{i, j}}-x_{i-1 / 2}+x_{j}\right) \\
& -\frac{1}{2} \sum_{j=1}^{i-1} n\left(t, x_{j+1}\right) \Delta x_{j+1} f\left(x_{i+1 / 2}-x_{j+1}, x_{j+1}\right)\left(x_{l_{i+1, j+1}+\frac{1}{2} \gamma_{i+1, j+1}}-x_{i+1 / 2}+x_{j+1}\right) \\
& +\mathcal{O}\left(\Delta x^{2}\right) .
\end{aligned}
$$

Approximating the function $x \mapsto n(t, x) f\left(x_{i \pm 1 / 2}-x, x\right)$ at $x_{j}$ by $n(t, x) f\left(x_{i \pm 1 / 2}-x, x\right)$ evaluated at $x=x_{j+1}$ in the first term, we evaluate

$$
\begin{aligned}
E_{1}= & \frac{1}{2} \sum_{j=1}^{i-1}\left\{\Delta x_{j}\left(x_{l_{i, j}+\frac{1}{2} \gamma_{i, j}}-x_{i-1 / 2}+x_{j}\right)-\Delta x_{j+1}\left(x_{l_{i+1, j+1}+\frac{1}{2} \gamma_{i+1, j+1}}-x_{i+1 / 2}+x_{j+1}\right)\right\} \\
& \times n\left(t, x_{j+1}\right) f\left(x_{i+1 / 2}-x_{j+1}, x_{j+1}\right)+\mathcal{O}\left(\Delta x^{2}\right) .
\end{aligned}
$$


By using the identities in the beginning of this section, we calculate

$$
\begin{aligned}
\Delta x_{j} & \left(x_{l_{i, j}+\frac{1}{2} \gamma_{i, j}}-x_{i-1 / 2}+x_{j}\right)-\Delta x_{j+1}\left(x_{l_{i+1, j+1}+\frac{1}{2} \gamma_{i+1, j+1}}-x_{i+1 / 2}+x_{j+1}\right) \\
= & \left(\Delta x_{j}-\Delta x_{j+1}\right)\left(x_{l_{i, j}+\frac{1}{2} \gamma_{i, j}}-x_{i-1 / 2}+x_{j}\right) \\
& -\Delta x_{j+1}\left[\left(x_{l_{i+1, j+1}+\frac{1}{2} \gamma_{i+1, j+1}}-x_{i+1 / 2}+x_{j+1}\right)-\left(x_{l_{i, j}+\frac{1}{2} \gamma_{i, j}}-x_{i-1 / 2}+x_{j}\right)\right] \\
= & \mathcal{O}\left(h^{2}\right)\left\{h_{1} g^{\prime}\left(\xi_{22}\right)+\mathcal{O}\left(h^{2}\right)\right\}-\left\{h g^{\prime}\left(\xi_{j+1}\right)+\mathcal{O}\left(h^{3}\right)\right\}\left[h_{1}\left\{g^{\prime}\left(\xi_{12}\right)-g^{\prime}\left(\xi_{22}\right)\right\}+\mathcal{O}\left(h^{2}\right)\right] \\
= & \mathcal{O}\left(h^{3}\right)-\mathcal{O}(h)\left[h_{1} h g^{\prime}\left(\xi_{22}\right)+\mathcal{O}\left(h^{2}\right)\right] \\
= & \mathcal{O}\left(h^{3}\right) .
\end{aligned}
$$

Therefore, substituting (3.30) in (3.29), we obtain

$$
E_{1}=\mathcal{O}\left(\Delta x^{2}\right)
$$

Next, let us calculate $E_{3}$ defined in (3.20) as follows

$$
\begin{aligned}
& E_{3}=\sum_{j=1}^{i-1} N_{j}(t) \int_{x_{i-1 / 2}-x_{j}}^{x_{l_{i, j}+\frac{1}{2} \gamma_{i, j}}} \frac{\left(x-x_{i}+x_{j}\right)}{\Delta x_{i}+\Delta x_{i+1}} f\left(x, x_{j}\right) \mathrm{d} x \\
& +\sum_{j=1}^{i} N_{j}(t) \int_{x_{l_{i+1, j}+\frac{1}{2} \gamma_{i+1, j}}^{x_{i+1 / 2}-x_{j}}} \frac{\left(x-x_{i}+x_{j}\right)}{\Delta x_{i}+\Delta x_{i+1}} f\left(x, x_{j}\right) \mathrm{d} x \\
& -\sum_{j=1}^{i-2} N_{j}(t) \int_{x_{i-3 / 2}-x_{j}}^{x_{l_{i-1, j}+\frac{1}{2} \gamma_{i-1, j}}} \frac{\left(x-x_{i-1}+x_{j}\right)}{\Delta x_{i}+\Delta x_{i-1}} f\left(x, x_{j}\right) \mathrm{d} x \\
& -\sum_{j=1}^{i-1} N_{j}(t) \int_{x_{l_{i, j}+\frac{1}{2} \gamma_{i, j}}^{x_{i-1 / 2}-x_{j}}} \frac{\left(x-x_{i-1}+x_{j}\right)}{\Delta x_{i}+\Delta x_{i-1}} f\left(x, x_{j}\right) \mathrm{d} x .
\end{aligned}
$$

Applying the left rectangle rule to the integrals appearing in first and third terms, and the right rectangle rule to the integrals in second and fourth terms, we estimate

$$
\begin{aligned}
E_{3}= & -\frac{1}{2} \sum_{j=1}^{i-1} N_{j}(t) f\left(x_{i-1 / 2}-x_{j}, x_{j}\right) \frac{\Delta x_{i}}{\Delta x_{i}+\Delta x_{i+1}}\left(x_{l_{i, j}+\frac{1}{2} \gamma_{i, j}}-x_{i-1 / 2}+x_{j}\right) \\
& -\frac{1}{2} \sum_{j=1}^{i-1} N_{j}(t) f\left(x_{i+1 / 2}-x_{j}, x_{j}\right) \frac{\Delta x_{i}}{\Delta x_{i}+\Delta x_{i+1}}\left(x_{l_{i+1, j}+\frac{1}{2} \gamma_{i+1, j}}-x_{i+1 / 2}+x_{j}\right) \\
& +\frac{1}{2} \sum_{j=1}^{i-2} N_{j}(t) f\left(x_{i-3 / 2}-x_{j}, x_{j}\right) \frac{\Delta x_{i-1}}{\Delta x_{i}+\Delta x_{i-1}}\left(x_{l_{i-1, j}+\frac{1}{2} \gamma_{i-1, j}}-x_{i-3 / 2}+x_{j}\right) \\
& +\frac{1}{2} \sum_{j=1}^{i-1} N_{j}(t) f\left(x_{i-1 / 2}-x_{j}, x_{j}\right) \frac{\Delta x_{i-1}}{\Delta x_{i}+x_{i-1}}\left(x_{l_{i, j}+\frac{1}{2} \gamma_{i, j}}-x_{i-1 / 2}+x_{j}\right)+\mathcal{O}\left(\Delta x^{2}\right) .
\end{aligned}
$$

Let us approximate $f$ at $\left(x_{i-3 / 2}-x_{j}, x_{j}\right)$ by $f$ expanded around $\left(x_{i-1 / 2}-x_{j}, x_{j}\right)$ in the third term and $f$ at $\left(x_{i-1 / 2}-x_{j}, x_{j}\right)$ by $f$ expanded around $\left(x_{i+1 / 2}-x_{j}, x_{j}\right)$ in the fourth term. Further, we replace $j$ by $j+1$ and 
$j-1$ respectively in second and third terms. Also, the relationship $N_{j}(t)=n\left(t, x_{j}\right) \Delta x_{j}+\mathcal{O}\left(\Delta x^{3}\right)$ is used to get

$$
\begin{aligned}
E_{3}= & -\frac{1}{2} \sum_{j=1}^{i-1} n\left(t, x_{j}\right) \Delta x_{j} f\left(x_{i-1 / 2}-x_{j}, x_{j}\right) \frac{\Delta x_{i}}{\Delta x_{i}+\Delta x_{i+1}}\left(x_{l_{i, j}+\frac{1}{2} \gamma_{i, j}}-x_{i-1 / 2}+x_{j}\right) \\
& -\frac{1}{2} \sum_{j=0}^{i-2} n\left(t, x_{j+1}\right) \Delta x_{j+1} f\left(x_{i+1 / 2}-x_{j+1}, x_{j+1}\right) \\
& \times \frac{\Delta x_{i}}{\Delta x_{i}+\Delta x_{i+1}}\left(x_{l_{i+1, j+1}+\frac{1}{2} \gamma_{i+1, j+1}}-x_{i+1 / 2}+x_{j+1}\right) \\
& +\frac{1}{2} \sum_{j=2}^{i-1} n\left(t, x_{j-1}\right) \Delta x_{j-1} f\left(x_{i-1 / 2}-x_{j-1}, x_{j-1}\right) \\
& \times \frac{\Delta x_{i-1}}{\Delta x_{i}+\Delta x_{i-1}}\left(x_{l_{i-1, j-1}+\frac{1}{2} \gamma_{i-1, j-1}}-x_{i-3 / 2}+x_{j-1}\right) \\
& +\frac{1}{2} \sum_{j=1}^{i-2} n\left(t, x_{j}\right) \Delta x_{j} f\left(x_{i+1 / 2}-x_{j}, x_{j}\right) \frac{\Delta x_{i-1}}{\Delta x_{i}+x_{i-1}}\left(x_{l_{i, j}+\frac{1}{2} \gamma_{i, j}}-x_{i-1 / 2}+x_{j}\right) \\
& +\mathcal{O}\left(\Delta x^{2}\right) .
\end{aligned}
$$

Without loss of generality, we can drop the terms which are second order accurate. Moreover, we approximate the functions $x \mapsto n(t, x) f\left(x_{i \mp 1 / 2}-x, x\right)$ at point $x_{j}$ by $n(t, x) f\left(x_{i \mp 1 / 2}-x, x\right)$ evaluated at points $x=x_{j \mp 1}$ of the first and fourth terms, respectively, to obtain

$$
\begin{aligned}
E_{3}= & -\frac{1}{2} \sum_{j=2}^{i-1} n\left(t, x_{j-1}\right) f\left(x_{i-1 / 2}-x_{j-1}, x_{j-1}\right) \frac{\Delta x_{i} \Delta x_{j}}{\Delta x_{i}+\Delta x_{i+1}}\left(x_{l_{i, j}+\frac{1}{2} \gamma_{i, j}}-x_{i-1 / 2}+x_{j}\right) \\
& -\frac{1}{2} \sum_{j=1}^{i-2} n\left(t, x_{j+1}\right) f\left(x_{i+1 / 2}-x_{j+1}, x_{j+1}\right) \\
& \times \frac{\Delta x_{i} \Delta x_{j+1}}{\Delta x_{i}+\Delta x_{i+1}}\left(x_{l_{i+1, j+1}+\frac{1}{2} \gamma_{i+1, j+1}}-x_{i+1 / 2}+x_{j+1}\right) \\
& +\frac{1}{2} \sum_{j=2}^{i-1} n\left(t, x_{j-1}\right) f\left(x_{i-1 / 2}-x_{j-1}, x_{j-1}\right) \\
& \times \frac{\Delta x_{i-1} \Delta x_{j-1}}{\Delta x_{i}+\Delta x_{i-1}}\left(x_{l_{i-1, j-1}+\frac{1}{2} \gamma_{i-1, j-1}}-x_{i-3 / 2}+x_{j-1}\right) \\
& +\frac{1}{2} \sum_{j=1}^{i-2} n\left(t, x_{j+1}\right) f\left(x_{i+1 / 2}-x_{j+1}, x_{j+1}\right) \frac{\Delta x_{i-1} \Delta x_{j}}{\Delta x_{i}+x_{i-1}}\left(x_{l_{i, j}+\frac{1}{2} \gamma_{i, j}}-x_{i-1 / 2}+x_{j}\right) \\
& +\mathcal{O}\left(\Delta x^{2}\right) .
\end{aligned}
$$

Let us denote each summation with the factor $\frac{1}{2}$ on the right-hand side by $E_{11}, \ldots, E_{14}$ respectively. Therefore, we can write

$$
E_{3}=\left(E_{33}-E_{31}\right)+\left(E_{34}-E_{32}\right)+\mathcal{O}\left(\Delta x^{2}\right)
$$


To simplify (3.32), we first calculate $E_{33}-E_{31}$ as follows

$$
\begin{aligned}
E_{33}-E_{31}= & \frac{1}{2} \sum_{j=2}^{i-1} n\left(t, x_{j-1}\right) f\left(x_{i-1 / 2}-x_{j-1}, x_{j-1}\right) \\
& \times\left\{\frac{\Delta x_{i-1} \Delta x_{j-1}}{\Delta x_{i}+\Delta x_{i-1}}\left(x_{l_{i-1, j-1}+\frac{1}{2} \gamma_{i-1, j-1}}-x_{i-3 / 2}+x_{j-1}\right)\right. \\
& \left.-\frac{\Delta x_{i} \Delta x_{j}}{\Delta x_{i}+\Delta x_{i+1}}\left(x_{l_{i, j}+\frac{1}{2} \gamma_{i, j}}-x_{i-1 / 2}+x_{j}\right)\right\} .
\end{aligned}
$$

Again by using the identities mentioned in the beginning of this section, we need to estimate the following term for solving (3.33).

$$
\begin{aligned}
\Delta x_{i-1} \Delta x_{j-1}( & \left.\Delta x_{i}+\Delta x_{i+1}\right)\left(x_{l_{i-1, j-1}+\frac{1}{2} \gamma_{i-1, j-1}}-x_{i-3 / 2}+x_{j-1}\right) \\
& -\Delta x_{i} \Delta x_{j}\left(\Delta x_{i}+\Delta x_{i-1}\right)\left(x_{l_{i, j}+\frac{1}{2} \gamma_{i, j}}-x_{i-1 / 2}+x_{j}\right) \\
= & \Delta x_{i-1} \underbrace{\left(\Delta x_{j-1}-\Delta x_{j}\right)}_{=\mathcal{O}\left(h^{2}\right)}\left(\Delta x_{i}+\Delta x_{i+1}\right)\left(x_{l_{i-1, j-1}+\frac{1}{2} \gamma_{i-1, j-1}}-x_{i-3 / 2}+x_{j-1}\right) \\
& +\Delta x_{j}\left[\Delta x_{i-1}\left(\Delta x_{i}+\Delta x_{i+1}\right)\left(x_{l_{i-1, j-1}+\frac{1}{2} \gamma_{i-1, j-1}}-x_{i-3 / 2}+x_{j-1}\right)\right. \\
& \left.-\Delta x_{i}\left(\Delta x_{i}+\Delta x_{i-1}\right)\left(x_{l_{i, j}+\frac{1}{2} \gamma_{i, j}}-x_{i-1 / 2}+x_{j}\right)\right] \\
= & \mathcal{O}\left(h^{5}\right)+\left(h g^{\prime}\left(\xi_{j}\right)+\mathcal{O}\left(h^{3}\right)\right)[\underbrace{h g^{\prime}\left(\xi_{i-1}\right)}_{=h g^{\prime}\left(\xi_{i}\right)+\mathcal{O}\left(h^{2}\right)}+\mathcal{O}\left(h^{3}\right))\left(2 h g^{\prime}\left(\xi_{i}\right)+\mathcal{O}\left(h^{2}\right)\right) \\
& \left.\times\left(h_{1} g^{\prime}\left(\xi_{32}\right)+\mathcal{O}\left(h^{2}\right)\right)-\left(h g^{\prime}\left(\xi_{i}\right)+\mathcal{O}\left(h^{3}\right)\right)\left(2 h g^{\prime}\left(\xi_{i}\right)+\mathcal{O}\left(h^{2}\right)\right)\left(h_{1} g^{\prime}\left(\xi_{22}\right)+\mathcal{O}\left(h^{2}\right)\right)\right] \\
= & \mathcal{O}(h)\left[2 h^{2}\left(g^{\prime}\left(\xi_{i}\right)\right)^{2} h_{1}\left\{g^{\prime}\left(\xi_{32}\right)-g^{\prime}\left(\xi_{22}\right)\right\}\right]+\mathcal{O}\left(h^{5}\right) \\
= & \mathcal{O}(h) \cdot 2 h^{3} h_{1}\left(g^{\prime}\left(\xi_{i}\right)\right)^{2} g^{\prime}\left(\xi_{32}\right)+\mathcal{O}\left(h^{5}\right) \\
= & \mathcal{O}\left(h^{5}\right) .
\end{aligned}
$$

Inserting this estimate in (3.33), we obtain

$$
E_{33}-E_{31}=\mathcal{O}\left(h^{2}\right)
$$

Analogous to (3.34), we can easily show that

$$
E_{34}-E_{32}=\mathcal{O}\left(h^{2}\right) .
$$

Finally, substituting (3.34) and (3.35) into (3.32), we have

$$
E_{3}=\mathcal{O}\left(\Delta x^{2}\right) .
$$

In a similar way, we can prove that

$$
E_{3}^{\prime}=\mathcal{O}\left(\Delta x^{2}\right)
$$


Next, it can be easily observed from (3.20) and (3.21) that the error terms $E_{2}, E_{2}^{\prime}, E_{4}$ and $E_{4}^{\prime}$ are second order accurate independent of meshes. Therefore, by substituting (3.31), (3.36) and (3.37) into (3.24), we have

$$
\sigma_{i}(t)=\mathcal{O}\left(\Delta x^{2}\right) \text { if } i \in \mathfrak{A}_{1}, \mathfrak{A}_{2}, \mathfrak{A}_{3} .
$$

Thus, using (3.25), we obtain

$$
\|\sigma(t)\|=\mathcal{O}(\Delta x)
$$

This shows that the cell average technique is first order consistent on such type of non-uniform smooth grids.

Remark 3.1. It should be pointed out that, due to the cancellation of second order terms, the error terms $E_{2}, E_{2}^{\prime}, E_{3}, E_{3}^{\prime}, E_{4}$ and $E_{4}^{\prime}$ can be shown third order accurate on geometric grids. However, since this will not improve the order of consistency (because $E_{1}$ is only second order accurate for such grids), we do not include further calculations.

\section{Lipschitz Conditions on $\hat{\mathbf{B}}(\mathbf{N}(\mathrm{t}))$ and $\hat{\mathbf{D}}(\mathbf{N}(\mathrm{t}))$}

Let us consider the birth term for $0 \leq t \leq T$ and for all $\mathbf{N}, \hat{\mathbf{N}} \in \mathbb{R}^{I}$. We get from (2.15)

$$
\begin{aligned}
\|\hat{\mathbf{B}}(\mathbf{N})-\hat{\mathbf{B}}(\hat{\mathbf{N}})\| \leq & \sum_{i=1}^{I} \lambda_{i}^{-}\left(\bar{v}_{i-1}\right) H\left(\bar{v}_{i-1}-x_{i-1}\right)\left|\hat{B}_{i-1}(\mathbf{N})-\hat{B}_{i-1}(\hat{\mathbf{N}})\right| \\
& +\sum_{i=1}^{I}\left[\lambda_{i}^{+}\left(\bar{v}_{i}\right) H\left(\bar{v}_{i}-x_{i}\right)+\lambda_{i}^{-}\left(\bar{v}_{i}\right) H\left(x_{i}-\bar{v}_{i}\right)\right]\left|\hat{B}_{i}(\mathbf{N})-\hat{B}_{i}(\hat{\mathbf{N}})\right| \\
& +\sum_{i=1}^{I} \lambda_{i}^{+}\left(\bar{v}_{i+1}\right) H\left(x_{i+1}-\bar{v}_{i+1}\right)\left|\hat{B}_{i+1}(\mathbf{N})-\hat{B}_{i+1}(\hat{\mathbf{N}})\right| .
\end{aligned}
$$

The definitions of $\lambda_{i}^{ \pm}(x)$ and $H(x)$ in (2.14) and (2.13), respectively, guarantee that $0 \leq \lambda_{i}^{ \pm}(x) H(x) \leq 1$. Thus, by using this upper bound, the above inequality becomes

$$
\begin{aligned}
\|\hat{\mathbf{B}}(\mathbf{N})-\hat{\mathbf{B}}(\hat{\mathbf{N}})\| \leq & \sum_{i=1}^{I}\left|\hat{B}_{i-1}(\mathbf{N})-\hat{B}_{i-1}(\hat{\mathbf{N}})\right|+\sum_{i=1}^{I}\left|\hat{B}_{i}(\mathbf{N})-\hat{B}_{i}(\hat{\mathbf{N}})\right| \\
& +\sum_{i=1}^{I}\left|\hat{B}_{i+1}(\mathbf{N})-\hat{B}_{i+1}(\hat{\mathbf{N}})\right| .
\end{aligned}
$$

Substituting (2.6) into (4.1) and using the assumpution on $\beta$ defined in (2.18), we have

$$
\begin{aligned}
\|\hat{\mathbf{B}}(\mathbf{N})-\hat{\mathbf{B}}(\hat{\mathbf{N}})\| \leq & \frac{1}{2}\|\beta\|_{L^{\infty}} \sum_{i=1}^{I} \sum_{j=1}^{i-1} \sum_{x_{i-3 / 2} \leq x_{j}+x_{k}<x_{i-1 / 2}}\left|N_{j}(t) N_{k}(t)-\hat{N}_{j}(t) \hat{N}_{k}(t)\right| \\
& +\frac{1}{2}\|\beta\|_{L^{\infty}} \sum_{i=1}^{I} \sum_{j=1}^{i} \sum_{x_{i-1 / 2} \leq x_{j}+x_{k}<x_{i+1 / 2}}\left|N_{j}(t) N_{k}(t)-\hat{N}_{j}(t) \hat{N}_{k}(t)\right| \\
& +\frac{1}{2}\|\beta\|_{L^{\infty}} \sum_{i=1}^{I} \sum_{j=1}^{i+1} \sum_{x_{i+1 / 2} \leq x_{j}+x_{k}<x_{i+3 / 2}}\left|N_{j}(t) N_{k}(t)-\hat{N}_{j}(t) \hat{N}_{k}(t)\right| \\
\leq & \frac{3}{2}\|\beta\|_{L^{\infty}} \sum_{j=1}^{I} \sum_{k=1}^{I}\left|N_{j}(t) N_{k}(t)-\hat{N}_{j}(t) \hat{N}_{k}(t)\right| .
\end{aligned}
$$


Now we apply the following useful equality $N_{j}(t) N_{k}(t)-\hat{N}_{j}(t) \hat{N}_{k}(t)=\frac{1}{2}\left[\left(N_{j}(t)+\hat{N}_{j}(t)\right)\left(N_{k}(t)-\hat{N}_{k}(t)\right)+\right.$ $\left.\left(N_{j}(t)-\hat{N}_{j}(t)\right)\left(N_{k}(t)+\hat{N}_{k}(t)\right)\right]$ to get

$$
\begin{aligned}
\|\hat{\mathbf{B}}(\mathbf{N})-\hat{\mathbf{B}}(\hat{\mathbf{N}})\| \leq & \frac{3}{4}\|\beta\|_{L^{\infty}} \\
& \times \sum_{j=1}^{I} \sum_{k=1}^{I}\left[\left|\left(N_{j}(t)+\hat{N}_{j}(t)\right)\left\|\left(N_{k}(t)-\hat{N}_{k}(t)\right)|+|\left(N_{j}(t)-\hat{N}_{j}(t)\right)\right\|\left(N_{k}(t)+\hat{N}_{k}(t)\right)\right|\right] .
\end{aligned}
$$

It can be easily shown that the total number of particles decreases in a coagulation process, i.e.

$$
\sum_{j=1}^{I} N_{j}(t) \leq N_{T}^{0}:=\text { Total number of particles which are taken initially. }
$$

The equation (4.2) can be rewritten as

$$
\begin{aligned}
\|\hat{\mathbf{B}}(\mathbf{N})-\hat{\mathbf{B}}(\hat{\mathbf{N}})\| & \leq \frac{3}{2} N_{T}^{0}\|\beta\|_{L^{\infty}}\left[\sum_{k=1}^{I}\left|\left(N_{k}(t)-\hat{N}_{k}(t)\right)\right|+\sum_{j=1}^{I}\left|\left(N_{j}(t)-\hat{N}_{j}(t)\right)\right|\right] \\
& \leq C\|\mathbf{N}-\hat{\mathbf{N}}\|,
\end{aligned}
$$

where $C:=3 N_{T}^{0}\|\beta\|_{L^{\infty}}$. Similarly as before we can easily show the Lipschitz condition for death term as

$$
\|\hat{\mathbf{D}}(\mathbf{N})-\hat{\mathbf{D}}(\hat{\mathbf{N}})\| \leq C\|\mathbf{N}-\hat{\mathbf{N}}\| .
$$

Thus, Theorem 2.5 completes the proof of Theorem 2.6 .

\section{Conclusions}

We have presented a detailed convergence analysis of the cell average technique for nonlinear continuous Smoluchowski coagulation equation. It is proved that the cell average technique is second order convergent on uniform grids. However, it gives only a first order convergence on non-uniform smooth geometric grids. In order to obtain a second order convergence on geometric grids, either one needs to adapt a different approach than the one presented here, or modify the error term $E_{1}$ which may lead to some improvements in the CAT. It is also interesting to analyze CAT for nonlinear continuous SCE on more general grids, which we intend to study in future.

Acknowledgements. The authors gratefully acknowledges very helpful comments of a referee which led to some significant improvements in the paper.

\section{REFERENCES}

[1] J.P. Bourgade and F. Filbert, Convergence of a finite volume scheme for coagulation-fragmentation equations. Math. Comput. 77 (2008) 851-882.

[2] P.B. Dubovskii, Mathematical Theory of Coagulation. In vol. 23 of Lecture notes. Global Analysis Research Center, Seoul National university (1994).

[3] P.B. Dubovskii, V.A. Galkin and I.W. Stewart, Exact solutions for the coagulation-fragmentation equations. J. Phys. A: Math. Gen. 25 1992) 4737-4744.

[4] P.B. Dubovskii and I.W. Stewart, Existence, uniqueness and mass conservation for the coagulation-fragmentation equation. Math. Meth. Appl. Sci. 19 (1996) 571-591.

[5] A. Eibeck and W. Wagner, An efficient stochastic algorithm for studying coagulation dynamics and gelation phenomena. SIAM J. Sci. Comput. 22 (2000) 802-821. 
[6] A. Eibeck and W. Wagner, Stochastic particle approximations for Smoluchowski's coagulation equation. Ann. Appl. Probab. 11 (2001) 1137-1165.

[7] M. Escobedo, P. Laurençot, S. Mischler and B. Perthame, Gelation and mass conservation in coagulation-fragmentation models. J. Differ. Equ. 195 (2003) 143-174.

[8] R.C. Everson, D. Eyre and Q.P. Campbell, Spline method for solving continuous batch grinding and similarity equations. Comput. Chem. Eng. 21 (1997) 1433-1440.

[9] F. Filbet and P. Laurençot, Mass-conserving solutions and non-conservative approximation to the smoluchowski coagulation equation. Arch. Math. 83 (2004) 558-567.

[10] F. Filbet and P. Laurençot, Numerical simulation of the Smoluchowski coagulation equation. SIAM J. Sci. Comput. 25 (2004) $2004-2028$.

[11] N. Fournier and P. Laurençot, Existence of self-similar solutions to Smoluchowski's coagulation equation. Commun. Math. Phys. 256 (2005) 589-609.

[12] N. Fournier and P. Laurençot, Well-posedness of Smoluchowski's coagulation equation for a class of homogeneous kernels. J. Funct. Anal. 233 (2006) 351-379.

[13] A.K. Giri, Mathematical and numerical analysis for coagulation-fragmentation equations. Ph.D. Thesis. Otto-von-GuerickeUniversity Magdeburg, Germany, 2010.

[14] A.K. Giri and E. Hausenblas, Convergence analysis of sectional methods for solving aggregation population balance equations: The fixed pivot technique. Nonlinear Anal. Real World Appl. 14 (2013) 2068-2090.

[15] A.K. Giri, J. Kumar and G. Warnecke, The continuous coagulation equation with multiple fragmentation. J. Math. Anal. Appl. 374 (2011) 71-87.

[16] A.K. Giri, Ph. Laurençot and G. Warnecke, Weak solutions to the continuous coagulation equation with multiple fragmentation. Nonlin. Anal. 75 (2012) 2199-2208.

[17] A.K. Giri and G. Warnecke, Uniqueness for the continuous coagulation-fragmentation equation with strong fragmentation. $Z$. Angew. Math. Phys. 62 (2011) 1047-1063.

[18] W. Hundsdorfer and J.G. Verwer, Numerical solution of time-dependent advection-diffusion-reaction equations. Springer-Verlag New York, USA, 1st edition (2003).

[19] J. Kumar, M. Peglow, G. Warnecke and S. Heinrich, An efficient numerical technique for solving population balance equation involving aggregation, breakage, growth and nucleation. Powder Technol. 179 (2007) 205-228.

[20] J. Kumar, M. Peglow, G. Warnecke, S. Heinrich and L. Mörl, Improved accuracy and convergence of discretized population balance for aggregation: The cell average technique. Chem. Eng. Sci. 61 (2006) 3327-3342.

[21] S. Kumar and D. Ramkrishna, On the solution of population balance equations by discretization-I. A fixed pivot technique. Chem. Eng. Sci. 51 (1996) 1311-1332.

[22] S. Kumar and D. Ramkrishna, On the solution of population balance equations by discretization II. A moving pivot technique. Chem. Eng. Sci. 51 (1996) 1333-1342.

[23] J. Kumar and G. Warnecke, Convergence analysis of sectional methods for solving breakage population balance equations - I: The fixed pivot technique. Numer. Math. 111 (2008) 81-108.

[24] J. Kumar and G. Warnecke, Convergence analysis of sectional methods for solving breakage population balance equations - II: The cell average technique. Numer. Math. 110 (2008) 539-559.

[25] W. Lamb, Existence and uniqueness results for the continuous coagulation and fragmentation equation. Math. Meth. Appl. Sci. 27 (2004) 703-721.

[26] P. Laurençot, On a class of continuous coagulation- fragmentation equations. J. Differ. Equ. 167 (2000) $245-274$

[27] P. Laurençot and S. Mischler, From the discrete to the continuous coagulation-fragmentation equations. Proc. Roy. Soc. Edinburgh 132A (2002) 1219-1248.

[28] M.H. Lee, On the validity of the coagulation equation and the nature of runaway growth. Icarus 143 (2000) 74-86.

[29] P. Linz, Convergence of a discretization method for integro-differential equations. Numer. Math. 25 (1975) $103-107$.

[30] D.J. McLaughlin, W. Lamb and A.C. McBride, A semigroup approach to fragmentation models. SIAM J. Math. Anal. 28 (1997) 1158-1172.

[31] D.J. McLaughlin, W. Lamb and A.C. McBride, An existence and uniqueness result for a coagulation and multiple-fragmentation equation. SIAM J. Math. Anal. 28 (1997) 1173-1190.

[32] M. Nicmanis and M.J. Hounslow, Finite-element methods for steady-state population balance equations. AICHE J. 44 (1998) $2258-2272$

[33] S. Rigopoulos and A.G. Jones, Finite-element scheme for solution of the dynamic population balance equation. AICHE J. 49 (2003) 1127-1139.

[34] J. Su, Z. Gu, Y. Li, S. Feng and X.Y. Xu, Solution of population balance equation using quadrature method of moments with an adjustable factor. Chem. Eng. Sci. 62 (2007) 5897-5911.

[35] I.W. Stewart, A global existence theorem for the general coagulation-fragmentation equation with unbounded kernels. Math. Meth. Appl. Sci. 11 (1989) 627-648. 